\title{
ON THE UNIQUENESS OF SINGULAR SOLUTIONS TO BOUNDARY -INITIAL VALUE PROBLEMS IN LINEAR ELASTODYNAMICS
}

Thesis by

G. Samuel Brockway

In Partial Fulfillment of the Requirements

For the Degree of

Doctor of Philosophy

California Institute of Technology

Pasadena, California

1972

(Submitted May 19, 1972) 
-ii-

To Patsy 


\section{Acknowledgments}

I am deeply grateful to Professor Eli Sternberg for his counsel throughout my studies at Caltech. I am especially indebted to him for his guidance during the course of my research and his substantial aid in the preparation of the manuscript. Professor James Knowles' invaluable suggestions and criticisms are also sincerely appreciated.

Special recognition is due Mrs. Julie Powell for her careful typing of this thesis and my wife Pat for her patient proof-reading of the manuscript. In addition, I wish to thank the National Science Foundation, the Office of Naval Research, and the Shell Companies Foundation for their financial support during my graduate training. 


\section{Abstract}

This investigation deals with certain generalizations of the classical uniqueness theorem for the second boundary-initial value problem in the linearized dynamical theory of not necessarily homogeneous nor isotropic elastic solids. First, the regularity assumptions underlying the foregoing theorem are relaxed by admitting stress fields with suitably restricted finite jump discontinuities. Such singularities are familiar from known solutions to dynamical elasticity problems involving discontinuous surface tractions or non-matching boundary and initial conditions. The proof of the appropriate uniqueness theorem given here rests on a generalization of the usual energy identity to the class of singular elastodynamic fields under consideration.

Following this extension of the conventional uniqueness theorem, we turn to a further relaxation of the customary smoothness hypotheses and allow the displacement field to be differentiable merely in a generalized sense, thereby admitting stress fields with squareintegrable unbounded local singularities, such as those encountered in the presence of focusing of elastic waves. A statement of the traction problem applicable in these pathological circumstances necessitates the introduction of "weak solutions"' to the field equations that are accompanied by correspondingly weakened boundary and initial conditions. A uniqueness theorem pertaining to this weak formulation 
is then proved through an adaptation of an argument used by O. Ladyzhenskaya in connection with the first boundary-initial value problem for a second-order hyperbolic equation in a single dependent variable. Moreover, the second uniqueness theorem thus obtained contains, as a special case, a slight modification of the previously established uniqueness theorem covering solutions that exhibit only finite stress-discontinuities. 
- vi -

Table of Contents

$\underline{\text { Page }}$

Introduction .................. 1

1. Notation and mathematical preliminaries.

The classical uniqueness theorem for the

traction problem in linear elastodynamics ..... 6

2. Uniqueness of solution to the traction

problem for piecewise regular

elastodynamic states ................ 14

3. Uniqueness of solution to the traction

problem for weak elastodynamic states ....... 32

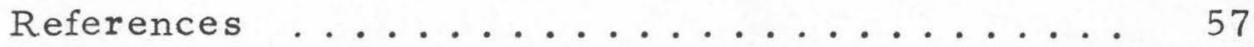


Introduction.

Rising interest in transient wave propagation during recent years has led to a host of new solutions to boundary-initial value problems in the linearized dynamical theory of elasticity. Many of these solutions, as well as some of those obtained long ago, exhibit finite jump discontinuities or even local infinities in the stress field as a consequence of discontinuous data, non-matching boundary and initial conditions, or as a result of focusing effects. On the other hand, solutions displaying such pathologies are clearly beyond the scope of Neumann's [1] classical uniqueness theorem, in which both the displacements and the stresses are assumed to be continuous functions of position and time. Indeed, the sense in which such singular elastodynamic fields "solve" the problems considered is in need of clarification. It is the purpose of this investigation to establish uniqueness theorems in linear elastodynamics, applicable to not necessarily homogeneous nor isotropic materials, which encompass singular problems of the type just alluded to and thus, at the same time, supply an appropriate formulation for such problems.

With regard to finite stress-discontinuities, we recall a well-known result from the dynamics of continuous media, which may roughly be stated as follows ${ }^{1}$ : if the stress field as sociated with the motion of a body undergoes a jump discontinuity across some (sufficiently smooth) propagating surface, while the displacement field

$\overline{1}$ See Truesdell and Toupin [2] (Section 205) or Gurtin [3] (Section 73); the latter treatment is confined to infinitesimal deformations. 
is continuous, then the principle of balance of linear momentum is equivalent to the stress equations of motion together with a familiar set of jump relations, provided the two fields are suitably regular away from the moving surface of discontinuity. Accordingly, any proper formulation of a boundary-initial value problem for linearized elastodynamics in the circumstances under consideration must include, directly or indirectly, the aforementioned jump conditions ${ }^{1}$.

In Section 2, having disposed of the necessary preliminaries and a review of the classical uniqueness theorem in Section 1, we deal with the uniqueness issue pertaining to the traction problem of elastodynamics in the presence of finite stress-discontinuities. Our main objective here is a uniqueness theorem covering a formulation of the problem that includes the stress equations of motion accompanied by the jump conditions, in addition to appropriate regularity hypotheses on the displacements and stresses. The proof of the uniqueness theorem given in this section is based on an extension of the usual energy identity to the class of singular fields at hand. There is no difficulty in using this energy identity to arrive at a generalization of the uniqueness theorem established in Section 2 to the corresponding mixed problem, which contains the first and second problems as special cases.

In this connection, it may be worth remarking that the appropriate stress-discontinuities at the wave fronts often appear to be inferred from the solution rather than explicitly assumed in the formulation of the problem. Nevertheless, careful scrutiny of the method used to deduce the solution (e.g., formal application of the Laplace transform) reveals that the relevant jump relations have in fact been tacitly assumed to hold. 
In Section 3, we turn to a further relaxation of the customary smoothness hypotheses underlying the conventional uniqueness theorem for the second boundary-initial value problem and permit the displacement field to possess merely generalized first derivatives in the sense of Sobolev [4], thereby admitting stress fields with isolated square-integrable infinities. A suitable statement of the traction problem in this context is obtained through the introduction of "weak solutions" to the field equations, along with correspondingly weakened boundary and initial conditions. A uniqueness theorem pertaining to such a weak formulation of the problem is then proved through an adaptation of an argument used by Ladyzhenskaya [5] in connection with the first boundary - initial value problem for a single, secondorder hyperbolic equation. Finally, the resulting uniqueness theorem is shown to yield, as a corollary, a modified version of the theorem proved in Section 2 .

It should be mentioned that Knops and Payne $[6]^{1}$ have previously established a uniqueness theorem for a class of weak solutions to the mixed problem of linear elastodynamics in the absence of any assumption regarding the form-definiteness of the elasticity tensor. The principal concern in [6] appears to be with weakening the hypotheses on the elasticity tensor in the classical theor $\mathrm{em}^{2}$ rather than those on the regularity of the stresses and displacements. The definition of a weak solution adopted in [6] contains, in addition to

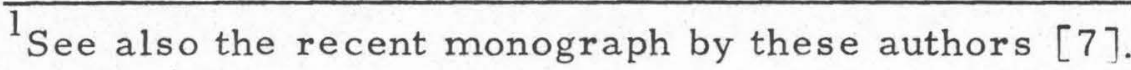

${ }^{2}$ With respect to this issue, see the earlier paper by Brun [87. 
generalizations of the field equations, boundary conditions and initial conditions, also an "energy conservation law" which has no place in a properly extended statement of the problem. In this regard, it is relevant to note that the omission of this conservation postulate from the definition of a weak solution given in [6] results in a formulation of the problem which remains equivalent to the classical formulation for elastodynamic fields having sufficient regularity. Moreover, if the elasticity tensor is assumed to be positive semi-definite and the extraneous question as to whether uniqueness persists in the absence of such a restriction is thereby set aside, uniqueness follows at once because of the inclusion of the conservation postulate among the hypotheses.

Working within the framework of the linearized dynamical theory of thermoelasticity, Dafermos [9] established the existence, uniqueness and asymptotic stability of "generalized solutions" to mixed boundary-initial value problems with homogeneous boundary conditions. The rather elaborate abstract function-analytic tools employed by Dafermos, although essential to his chief objective which concerns the existence and stability issues, are not necessary for the treatment of the uniqueness questions raised in the present paper. Nevertheless, the uniqueness argument used in [9] could be adapted to prove the uniqueness theorem presented in Section 3, albeit the proof given here appears to be more straightforward. Observe as well that our assumptions regarding the regularity of the underlying spatial domain are less restrictive than those introduced in [9]. 
Finally, mention should be made of a paper by Friedrichs [10] in which the existence and uniqueness of certain weak solutions to homogeneous boundary value problems for symmetric positive systems of first-order partial differential equations are established. Since the equations of linear elastodynamics can be cast into such a form and since Friedrichs' method can be modified to encompass boundaryinitial value problems, a theorem analogous to the one obtained in Section 3 could alternatively be based on Friedrichs' work. 
1. Notation and mathematical preliminaries. The classical uniqueness theorem for the traction problem in linear elastodynamics.

Throughout this investigation, lower case letters when not underscored represent scalars, while lower case letters underscored with a tilde denote tensors of positive order. We employ the usual indicial notation in connection with the Cartesian components of tensors; namely, Latin subscripts have the range $(1,2,3)$, summation over repeated indices being implied. In addition, we write $\underset{\sim}{\varrho} \mathcal{\chi}$ for the fully contracted outer product $\varepsilon_{i j} Y_{i j}$ of two second-order tensors $£, \mathcal{L}$ and define the norm $|\underset{\sim}{\omega}|$ of a tensor $\underset{w}{ }$ of arbitrary order to be the square root of the fully contracted outer product of $\underset{\sim}{w}$ with itself.

The symbol $\mathrm{E}_{\mathrm{n}}$ is used for $\mathrm{n}$-dimensional Euclidean space, except that we write $\mathrm{E}$ in place of $\mathrm{E}_{3}$. Moreover, the statement $(\underset{\sim}{x}, t) \in E_{4}$ is understood to signify $\left.{ }^{1} \underset{\sim}{x}, t\right) \in E x(-\infty, \infty)$. We mean by a domain in $E_{n}$ an open connected subset of $E_{n}$ and by a region a domain together with all, some or none of its boundary points. If $D$ is a subset of $E_{n}$, then $\stackrel{D}{D}, \bar{D}$ and $\partial \mathrm{D}$ denote the interior, closure and boundary of $D$, in that order. We shall say that a collection $\left\{D_{1}, D_{2}, \cdots, D_{m}\right\}$ of subsets of a domain $D$ in $E_{n}(n \geq 2)$ is a partition of $D$, provided that

$$
D_{i} \cap D_{j}=\phi(i \neq j ; i, j=1, \cdots, m) \text { and } \bigcup_{j=1}^{m} \bar{D}_{j}=\bar{D} \text {. }
$$

By a partition of an interval $\left(t_{1}, t_{2}\right)$, on the other hand, we mean a finite set of real numbers $\left\{\tau_{0}, \tau_{1}, \cdots, \tau_{m}\right\}$, such that

$$
t_{1}=\tau_{0}<\tau_{1}<\cdots<\tau_{m}=t_{2} .
$$

\footnotetext{
The conventional notation for the Cartesian product of two sets is used throughout this paper.
} 
As to smoothness classes of functions, if $D$ is a subset of $E_{n}$, then $C(D)$ denotes the collection of all tensor-valued functions that are continuous on D. Furthermore, if $\mathrm{m}$ is a positive integer, then a tensor-valued function is said to be in $C^{m}(D)$ if it is in $C(D)$ and all of its partial derivatives of order up to and including $\mathrm{m}$ exist on the interior of $D$ and coincide there with functions continuous on D. As is customary, we shall use the symbol $\mathrm{C}^{\infty}$ (D) for the class of functions that belong to $\mathrm{C}^{\mathrm{m}}$ (D) for every positive integer $\mathrm{m}$.

If $R$ is a region in $E, T$ an interval, and $\varphi$ a scalar field defined on $R \times T$ with values $\varphi(\underset{\sim}{x}, t)$ for each $(\underset{\sim}{x}, t) \in R \times T$, we let $\varphi(\cdot, t)$ for each $t \in T$ stand for the function on $R$ obtained by holding $t$ fixed, $\varphi(\underset{\sim}{x}, \cdot)$ for every $\underset{\sim}{x} \in R$ being defined analogously. As for the derivatives of any scalar function $\varphi$ defined on a subset $D$ of $E_{4}$, we write

$$
\begin{gathered}
\dot{\varphi}=\frac{\partial \varphi}{\partial t}, \ddot{\varphi}=\frac{\partial^{2} \varphi}{\partial t^{2}} \text { on } \mathrm{D}, \\
\varphi, \underbrace{i j \cdots k}_{\text {indic }}=\frac{\partial^{\mathrm{m}} \varphi}{\partial \mathrm{x}_{\mathrm{k}} \cdots \partial \mathrm{x}_{\mathrm{j}} \partial \mathrm{x}_{\mathrm{i}}} \text { on } \mathrm{D}(\mathrm{m}=1,2, \cdots),
\end{gathered}
$$

when the indicated partial differentiations are meaningful. In addition, if $\underset{\sim}{u}$ is a vector field on $D \subset E_{4}$, the symbol $\underset{\sim}{\sim}$ represents the secondorder tensor field with the Cartesian components $u_{i, j}$, while $\underset{\sim}{\dot{u}}$ denotes the vector field with the components $\dot{u}_{i}$. We adopt the latter convention for tensor fields of all orders.

By a regular region, we mean a bounded domain in $\mathrm{E}$, the boundary of which is the union of a finite collection of disjoint "closed 
regular surfaces", the latter term being used in the sense of Kellogg [11] (p. 112). The boundary of a regular region may have corners and edges. Moreover, as defined here, a regular region need not be simply connected ${ }^{l}$. If $\mathrm{R}$ is a regular region, then the regular part of $\partial R$, hereafter denoted by $\partial^{*}$, is the set of all points of $\partial \mathrm{R}$ at which a normal is defined. We recall that if $\mathrm{R}$ is a regular region and

$$
\underset{\sim}{f} \in C^{1}(R) \cap C(\bar{R})
$$

is a vector field whose divergence $\nabla \cdot \underset{\sim}{f}$ is integrable on $R$, then the divergence theorem applies ${ }^{2}$ to $\underset{\sim}{f}$ on $\mathrm{R}$; thus,

$$
\int_{\mathrm{R}} \nabla \cdot \underset{\sim \mathrm{f}}{\mathrm{f}} \mathrm{dV}=\int_{\sim} \underset{\sim}{\mathrm{n}} \cdot \underset{\sim}{\mathrm{f}} \mathrm{dA},
$$

where $\underset{\sim}{\mathrm{n}}$ is the unit outward normal of $\partial \mathrm{R}$.

We conclude this section by citing certain pertinent classical results from the linearized dynamical theory of elasticity. For this purpose we adopt ${ }^{3}$

Definition 1.1. (State; regular elastodynamic state). Let $\mathrm{R}$ be a bounded domain in $E,\left(0, t_{0}\right)$ a time-interval and $D \subset \bar{R} \times\left[0, t_{0}\right]$. Then, if $\underset{\sim}{\mathrm{u}}$ is a vector-valued and $\underset{\sim}{\sigma}$ a second-order tensor-valued function on $D$, the ordered pair $S=[\underset{\sim}{u}, \underset{\sim}{\sigma}]$ is called a state on $D$ with the displacement field $\underset{\sim}{u}$ and the stress field $\underset{\sim}{\sigma}$.

${ }^{1}$ Kellogg's regular region, in addition to being closed, has a boundary which is a single "closed regular surface".

2 This version of the divergence theorem follows easily from the broadest form proved by Kellogg [11] (p. 119).

${ }^{3} \mathrm{Cf}$., Wheeler and Sternberg, [12]. 
Suppose next that $\underset{\sim}{\sim}$ is a vector field on $D$, while $\rho$ and $\approx$ are, respectively, a scalar and fourth-order tensor field on $\bar{R}$. Then, $S=[\underset{\sim}{u}, \underset{\sim}{\sigma}]$ is said to be a regular elastodynamic state on $D$, corresponding to the body-force field $\underset{\sim}{f}$, the mass-density field $\rho$ and the

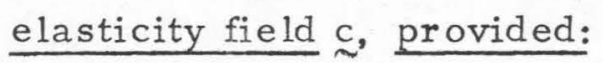

(i) $\underset{\sim}{f} \in \mathbb{C}(D) ; \rho \in C(\bar{R}), \quad \rho>0$ on $\bar{R} ; \stackrel{c}{\sim} \in \mathbb{C}(\bar{R})$ with

$$
c_{i j k \ell}=c_{j i k \ell}=c_{k \ell i j} \text { on } R
$$

and $\underset{\text { invertible }}{ }{ }^{1}$ on $\mathrm{R}$.

$$
\begin{aligned}
& \text { (ii) } \underset{\sim}{u} \in C^{2}(D) \cap C^{1}(D), \quad \sigma \in C^{1}(D) \cap C(D) . \\
& \text { (iii) } \sigma_{i j}=c_{i j k \ell} u_{k, \ell} \underline{\text { in }} \stackrel{\circ}{ } \\
& \sigma_{i j, j}+f_{i}=\rho \ddot{u}_{i} \underline{\text { in }} \stackrel{D}{ } .
\end{aligned}
$$

For the collection of all such states, we write

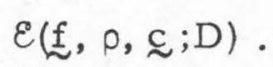

Equations (1.6) are the stress-displacement relations, and (1.7) are the stress equations of motion. In view of (1.6), the regularity assumptions (ii), although mutually consistent, are partly redundant. Equations (1.5) and (1.6), because of the assumed continuity of $\underset{\sim}{\sigma}$ on $\mathrm{D}$, assure the symmetry of $\underset{\sim}{\sigma}$ on $\mathrm{D}$.

We define equality and addition of states, as well as the multiplication of a state by a scalar, in the obvious manner. Furthermore, if $R$ is a regular region, $S=[\underset{\sim}{u}, \underset{\sim}{\sigma}]$ a state on $\bar{R} \times\left[0, t_{0}\right]$, and ${ }^{1}$ We say that $c$ is invertible on $R$ if for each $x \in R$ the determinant of the six-by-six coefficient matrix associated with the set of linear algebraic equations

$$
\alpha_{i j}=c_{i j k \ell}(x) \beta_{k \ell}
$$

is non-zero. 
$\underset{\sim}{n}$ the unit outward normal of $\partial R$, then the traction vector $\underset{\sim}{\mathrm{s}}$ of $S$ on $\partial R$ is characterized by

$$
s_{i}=\sigma_{i j} n_{j} \text { on } \ddot{*}^{*} R \times\left[0, t_{o}\right] \text {. }
$$

The traction problem for regular elastodynamic states (classical traction problem) can now be phrased economically, as follows. Given a regular region $\mathrm{R}$ and a time-interval $\left(0, \mathrm{t}_{0}\right)$, find

$$
S=[\underset{\sim}{u}, \underset{\sim}{\sigma}] \in \mathcal{E}\left(\underset{\sim}{f}, \rho, \underset{\sim}{c} ; \bar{R} \times\left[0, t_{0}\right]\right)
$$

for the data $\underset{\sim}{f}, \rho, \underset{\sim}{c}$ and subject to the initial conditions

$$
\underset{\sim}{\mathrm{u}}(\cdot, 0)=\underset{\sim}{\stackrel{\leftrightarrow}{u}}(\cdot, 0)=\stackrel{\circ}{\mathrm{v}} \text { on } \overline{\mathrm{R}}
$$

and the boundary condition

$$
\underset{\sim}{\mathrm{s}}=\stackrel{*}{\mathrm{*}} \text { on } \stackrel{*}{\partial} \mathrm{R} \times\left[0, \mathrm{t}_{\mathrm{o}}\right]
$$

where $\stackrel{\circ}{\sim}, \stackrel{\circ}{\mathrm{V}}$ and $\underset{\sim}{\stackrel{*}{\mathrm{~S}}}$ are prescribed functions.

A convenient version of the energy identity of linear elastodynamics is contained in

Theorem 1.1. (Classical energy identity). Let $\mathrm{R}$ be a regular region and

$$
S=[\underset{\sim}{u}, \underset{\sim}{\sigma}] \in \mathcal{E}\left(\underset{\sim}{f}, \rho, \underset{\sim}{c} ; \bar{R} \times\left[0, t_{0}\right]\right) .
$$

Then,

$\int_{0}^{t} \int_{R} \underset{\sim}{f} \cdot \underset{\sim}{\dot{u}} d V d \tau+\int_{0}^{t} \int_{\partial R} \underset{\sim}{s} \cdot \underset{\sim}{\dot{u}} d A d \tau=E(t)-E(0)$ for every $t \in\left[0, t_{0}\right]$,

where

${ }^{\mathrm{I}}$ Recall that $\partial \mathrm{k}$ is the regular part of $\partial \mathrm{R}$. 


$$
\left.\left.E(t)=\frac{1}{2} \int_{R} \rho(\underset{\sim}{x}) \dot{\sim}_{\sim}^{2}(\underset{\sim}{x}, t) d V+\frac{1}{2} \int_{R} \underset{\sim}{\sigma} \underset{\sim}{x}, t\right) \cdot \underset{\sim}{u} \underset{\sim}{x}, t\right) d V \text { for } \underline{\text { every }} t \in[0, t] .
$$

Observe that if $\underset{\sim}{\dddot{i}}$ and $\nabla \underset{\sim}{\mathfrak{i}}$ coincide on $\mathrm{R} \times\left(0, \mathrm{t}_{0}\right)$ with functions continuous on $\overline{\mathrm{R}} \times\left[0, t_{0}\right]$, one recovers from (1.11) the more conventional energy identity

$$
\int_{R}^{\underset{\sim}{f}}(\underset{\sim}{x}, t) \cdot \underset{\sim}{\stackrel{\dot{u}}{\sim}}(\underset{\sim}{x}, t) d V+\int_{\partial \mathrm{N}}^{\underset{\sim}{s}}(\underset{\sim}{x}, t) \cdot \underset{\sim}{\dot{u}}(\underset{\sim}{x}, t) d A=\dot{E}(t) \text { for every } t \in[0, t] .
$$

Since the argument traditionally used to deduce (1.13) cannot be applied to confirm (1.11) for elastodynamic fields of the regularity postulated here, we prove Theorem 1.1 instead by means of a modification of the method used by Wheeler and Sternberg [12] to establish a generalized energy identity of a different kind ${ }^{l}$.

Proof of Theorem 1.1. Choose $t \in\left(0, t_{0}\right), t_{1} \in(0, t)$ and define a vector field $\mathrm{p}$ on $\overline{\mathrm{R}}$ through

$$
\left.\left.\mathrm{p}_{\mathrm{j}} \underset{\sim}{\mathrm{x}}\right)=\int_{\mathrm{t}_{1}}^{\mathrm{t}} \sigma_{\mathrm{ij}}(\underset{\sim}{\mathrm{x}}, \tau) \dot{\mathrm{u}}_{\mathrm{i}} \underset{\sim}{\mathrm{x}}, \tau\right) \mathrm{d} \tau \text { for every } \underset{\sim}{\mathrm{x}} \in \overline{\mathrm{R}} .
$$

The hypotheses, Definition 1.1 and Equation (A) imply

$$
\underset{\sim}{\mathrm{p}} \in \mathrm{C}^{\mathrm{l}}(\mathrm{R}) \cap \mathrm{C}(\overline{\mathrm{R}}) \text {. }
$$

Next, compute the divergence of $\underset{\sim}{\mathrm{p}}$ and make use of (1.7), (1.6) and (1.5) to arrive at

${ }^{1}$ See also the extension to anisotropic elasticity by Wheeler [13]. The assumption that the second derivatives of the displacements possess limits as $t \rightarrow 0$, although adopted in [127 and [13], is omitted here. 


$$
\left.\left.\left.\nabla \cdot \underset{\sim}{p}(\underset{\sim}{\mathrm{x}})=-\int_{\mathrm{t}_{1}}^{\mathrm{t}} \underset{\sim}{\mathrm{f}} \underset{\sim}{\mathrm{x}}, \tau\right) \cdot \underset{\sim}{\operatorname{u}} \underset{\sim}{\mathrm{x}}, \tau\right) \mathrm{d} \tau+\int_{{ }_{1}}^{\mathrm{t}} \frac{\partial}{\partial \tau} \mathrm{e} \underset{\sim}{\mathrm{x}}, \tau\right) \mathrm{d} \tau \text { for all } \underset{\sim}{\mathrm{x}} \in \mathrm{R},
$$

where

$$
\mathrm{e}=\frac{1}{2} \rho \stackrel{\dot{u}}{\sim}^{2}+\frac{1}{2} \underset{\sim}{\sigma} \cdot \underset{\sim}{u} \text { on } \overline{\mathrm{R}} \times\left[0, \mathrm{t}_{0}\right]
$$

From (C) follows

$$
\nabla \cdot \underset{\sim}{\mathrm{p}}(\underset{\sim}{\mathrm{x}})=-\int_{\mathrm{t}_{1}}^{\mathrm{t}} \underset{\sim}{\mathrm{f}}(\underset{\sim}{\mathrm{x}}, \tau) \cdot \underset{\sim}{\dot{\mathrm{u}}}(\underset{\sim}{\mathrm{x}}, \tau) \mathrm{d} \tau+\mathrm{e}(\underset{\sim}{\mathrm{x}}, t)-e\left(\underset{\sim}{\mathrm{x}}, \mathrm{t}_{1}\right) \text { for all } \underset{\sim}{\mathrm{x}} \in R .
$$

Since from (D), (E) and Definition $1.1 \nabla \cdot p$ is continuous on $\bar{R},(B)$ and the hypothesis on $\mathrm{R}$ permit us to apply the divergence theorem to $\underset{\sim}{\mathrm{p}}$ on R. Doing so, taking (A), (E) into account and interchanging the order of integration, one obtains

$$
\int_{t_{1}}^{t} \int_{R}^{f} \underset{\sim}{f} \underset{\sim}{\dot{u}} d V d \tau+\int_{t_{1}}^{t} \int_{\partial R} \underset{\sim}{s} \cdot \underset{\sim}{\dot{u}} d A d \tau=E(t)-E\left(t_{1}\right),
$$

$E(t)$ being the total energy at the time $t$ (see (1.12)).

Finally, letting $t_{1} \rightarrow 0$ in $(F)$ yields (1.11) because of the arbitrariness of $t$ and the regularity assumptions (ii) in Definition 1.1. The proof is now complete.

The uniqueness of the solution to the classical traction problem, which was first proved by Neumann [1] with the aid of the classical energy identity, is asserted in

Theorem 1.2. (Neumann's classical uniqueness theorem). Let R be a $\underline{\text { regular region, and suppose: }}$ 
(a) $S^{\prime}=\left[\underset{\sim}{u_{\sim}^{\prime}}, \sigma_{\sim}^{\prime}\right] \in \mathcal{E}\left(\underset{\sim}{f}, \rho, \underset{\sim}{c} ; \bar{R} \times\left[0, t_{0}\right]\right)$,

$$
S^{\prime \prime}=\left[\underset{\sim}{u}, \sim_{\sim}^{\prime \prime}\right] \in \mathcal{E}\left(\underset{\sim}{f}, \rho, \underset{\sim}{\mathrm{c}} ; \overline{\mathrm{R}} \times\left[0, \mathrm{t}_{0}\right]\right) .
$$

$(\beta){\underset{\sim}{u}}^{\prime}(\cdot, 0)={\underset{\sim}{u}}^{\prime \prime}(\cdot, 0), \quad \stackrel{\sim}{\sim}^{\prime}(\cdot, 0)=\stackrel{\sim}{\sim}^{\prime \prime}(\cdot, 0)$ on $\bar{R}$

$$
\stackrel{s}{\prime}^{\prime}=\stackrel{s}{\sim}^{\prime \prime} \text { on }{ }^{*} \mathrm{R} \times\left[0, \mathrm{t}_{\mathrm{o}}\right] \text {. }
$$

Assume further that

(Y) $\underset{\sim}{\text { is positive semi-definite on }} \mathrm{R}$; $\underline{\text { i. e. }}$, suppose

$$
c_{i j k \ell} \stackrel{(x)}{\sim} Y_{i j} Y_{k \ell} \geq 0 \text { for every } \underset{\sim}{x} \in R
$$

and for every symmetric two-tensor $\underset{\sim}{\gamma}$. Then,

$$
S^{\prime}=S^{\prime \prime} \text { on } \bar{R} \times\left[0, t_{0}\right] \text {. }
$$

Brun [8] has shown that the conclusion in Theorem 1.2 remains valid even if hypothesis $(Y)$ is removed ${ }^{l}$.

${ }^{1}$ See also Knops and Payne [6], [7] (Section 8.3) and Gurtin [3] (Section 63). 
2. Uniqueness of solution to the traction problem for piecewise regular elastodynamic states.

In this section the statement of the traction problem of classical elastodynamics is generalized to encompass states that have continuous displacement fields but possess stress fields suffering finite jump discontinuities. Singular states of this type are shown to obey an analogue of the classical energy identity (Theorem 1.1), which is subsequently used to establish a corresponding extension of the classical uniqueness theorem (Theorem 1.2).

With a view toward formulating the traction problem in the presence of finite stress-discontinuities, we introduce the notion of a "smooth family of regions". This concept affords a convenient description of wave fronts, since the boundaries of the regions constituting such a family represent a surface deforming smoothly in time.

Definition 2.1. (Smooth and piecewise smooth families of regions). Let $R_{t}\left(t_{1} \leq t \leq t_{2}\right)$ be a one-parameter family of integrable subsets of $E$, each $\dot{R}_{t}\left(t_{1}<t<t_{2}\right)$ being a regular region. Suppose also that there exists a $\hat{t} \in\left(t_{1}, t_{2}\right)$ and a vector-valued function $\underset{\sim}{\varphi} \in \mathrm{C}^{2}\left(\overline{\mathrm{R}}_{\hat{\mathrm{t}}} \times\left[\mathrm{t}_{1}, \mathrm{t}_{2}\right]\right)$ such that the following conditions are met:

(i) For each $t$ in the closed interval $\left[t_{1}, t_{2}\right]$, the function $\underset{\sim}{\varphi}(\cdot, t)$ maps $\bar{R}_{\hat{t}}$ onto $\bar{R}_{t}$; i. e. , for every $\underset{\sim}{x} \in \bar{R}_{t}$ there exists a $\underset{\sim}{\in} \bar{R}_{\hat{t}}$ satisfying

$$
\underset{x}{x}=\mathscr{L}(\mathrm{y}, \mathrm{t}) .
$$

(ii) For each $t$ in the open interval $\left(t_{1}, t_{2}\right)$, the mapping $\varphi(\cdot, t)$ is one-to-one and has a positive Jacobian determinant on $\overline{\mathrm{R}}_{\hat{\mathrm{t}}}$, so that

$$
\left.\frac{\partial\left(\varphi_{1}, \varphi_{2}, \varphi_{3}\right)}{\partial\left(\mathrm{x}_{1}, \mathrm{x}_{2}, \mathrm{x}_{3}\right)}\right|_{(\mathrm{x}, \mathrm{t})}>0 \text { for every } \underset{\sim}{\mathrm{x}} \in \overline{\mathrm{R}}_{\hat{\mathrm{t}}} .
$$


Then, $R_{t}\left(t{ }_{1} \leq t \leq t_{2}\right)$ is said to be a smooth family of regions.

In addition, a one-parameter family $R_{t}\left(t_{1} \leq t \leq t_{2}\right)$ of subsets of $E$ will be called a piecewise smooth family of regions if there exists a partition $^{1}\left\{\tau_{0}, \tau_{1}, \cdots, \tau_{m}\right\}^{\text {of }}\left(t_{1}, t_{2}\right)$ such that $R_{t}\left(\tau_{k-1} \leq t \leq \tau_{k}\right)(k=1, \ldots, m)$ is a smooth family of regions.

Note that if $R_{t}\left(t_{1} \leq t \leq t_{2}\right)$ is a smooth family of regions, $R_{t_{1}}$ and $\mathrm{R}_{\mathrm{t}_{2}}$, although images of a regular region under the smooth mapping $\underset{\sim}{\varphi}(\cdot, t)$, need not themselves be regular regions, since $\underset{\sim}{\varphi}\left(\cdot, t_{1}\right)$ and $\underset{\sim}{\varphi}\left(\cdot, t_{2}\right)$ are not necessarily one-to-one. Indeed, ${\stackrel{\circ}{t_{1}}}_{1}$ and ${\stackrel{\circ}{\mathrm{t}_{2}}}_{2}$ may even be empty as exemplified by the two smooth families of regions

$$
\begin{aligned}
& R_{t}^{1}=\left\{\underset{\sim}{x} \in E \mid 0 \leq x_{\alpha} \leq 1 \quad(\alpha=1,2), \quad c t \leq x_{3} \leq 1\right\} \quad(0 \leq t \leq 1 / c), \\
& R_{t}^{2}=\left\{x_{\sim}^{x} \in E \mid 0 \leq x_{\alpha} \leq 1 \quad(\alpha=1,2), 2-c t \leq x_{3} \leq 1\right\} \quad(1 / c \leq t \leq 2 / c),
\end{aligned}
$$

where $c$ is a positive constant. Here, $\stackrel{\circ}{R}_{t}^{1}$ and $\stackrel{\circ}{t}_{t}^{2}$ are both void when $t=1 / c$. The family of sets $R_{t}(0 \leq t \leq 2 / c)$ given by

$$
R_{t}=R_{t}^{1} \text { for every } t \in[0,1 / c], R_{t}=R_{t}^{2} \text { for every } t \in(1 / c, 2 / c]
$$

constitutes a piecewise smooth family of regions, representing a family of collapsing and subsequently expanding rectangular parallelepipeds.

Let $R_{t}\left(t_{1} \leq t \leq t_{2}\right)$ be either a smooth or a piecewise smooth family of regions. We shall say that $R_{t}\left(t_{1} \leq t \leq t_{2}\right)$ sweeps out the open set $\mathrm{D}$ in $\mathrm{E}_{4}$ given by

$$
D=\left\{(\underset{\sim}{x}, t) \mid \underset{\sim}{x} \in \dot{R}_{t}, t \in\left(t_{1}, t_{2}\right)\right\} .
$$

Similarly, we call the surface $W$ in $E_{4}$ defined through

$$
W=\left\{(\underset{\sim}{x}, t) \mid \underset{\sim}{x} \in \partial R_{t}, t \in\left(t_{1}, t_{2}\right)\right\}
$$

${ }_{\text {See }(1.2) .}$ 
the surface swept out by $R_{t}\left(t_{1} \leq t \leq t_{2}\right)$.

Further, if $R_{t}\left(t_{1} \leq t \leq t_{2}\right)$ is a smooth family of regions, we use $\underset{\sim}{\mathrm{n}}(\cdot, \mathrm{t})$ to denote the unit outward normal of $\partial \mathrm{R}_{\mathrm{t}}$ and let

$$
\begin{aligned}
& \stackrel{*}{W}=\left\{(\underset{\sim}{x}, t) \mid \underset{\sim}{x} \in \hat{\sim}^{*} R_{t}, t \in\left(t_{1}, t_{2}\right)\right\}, \\
& x(\underset{\sim}{x}, t)=\underset{\sim}{n}(\underset{\sim}{x}, t) \cdot \frac{\partial \mathscr{\Psi}}{\partial t}\left(\mathscr{\sim}^{-1}(\underset{\sim}{x}, t), t\right) \text { for every }(\underset{\sim}{x}, t) \in W^{*}
\end{aligned}
$$

so that ${ }^{*}$ is the regular part of the surface W swept out by $R_{t}\left(t_{1} \leq t \leq t_{2}\right)$, and $x(\cdot, t)$, for every $t \in\left(t_{1}, t_{2}\right)$ is the normal component of the velocity of propagation of the surface $\partial R_{t}$. Next, we introduce the speed of propagation $c$ of the family $R_{t}\left(t_{1} \leq t \leq t_{2}\right)$ through

$$
c=|x| \text { on }{ }^{*}
$$

and the direction of propagation $\hat{\sim}$ of this family by means of

$$
\hat{\sim}=\operatorname{sgn}(x) \underset{\sim}{n} \text { on } N, N=\{(\underset{\sim}{x}, t) \in \underset{W}{W} \mid x(\underset{\sim}{x}, t) \neq 0\} \text {, }
$$

where $\operatorname{sgn}(\cdot)$ is the signum function. Accordingly, $c(\underset{\sim}{x}, t)$ is the speed at which a point $\underset{\sim}{\mathrm{x}}$ on the surface $\partial \mathrm{R}_{t}$ is moving in the direction of propagation $\underset{\sim}{\hat{n}}(x, t)$ at the time $t$. For a piecewise smooth family of regions $R_{t}\left(t \leq t \leq t_{2}\right)$, we use $\underset{\sim}{n}, x, c$, and $\underset{\sim}{\hat{\sim}}$ in the sense indicated above, bearing in mind that the regular part $\mathrm{W}_{\mathrm{W}}$ of $\mathrm{W}$ is now

$$
\stackrel{*}{W}=\left\{\left.(\underset{\sim}{x}, t)\right|_{\sim} ^{x} \in \stackrel{*}{*} R_{t}, \quad \underset{k=1}{m}\left(\tau_{k-1}, \tau_{k}\right)\right\},
$$

where $\left\{\tau_{0}, \tau_{1}, \cdots, \tau_{m}\right\}$ is the partition referred to in Definition 2.1.

The stress-discontinuities considered here will be assumed to occur across surfaces in $E_{4}$ which are swept out by piecewise smooth families of regions. Since in many cases of interest the re are several surfaces across which the stress field jumps, we introduce next 
Definition 2.2. (Regular partition of $R \times\left(0, t_{0}\right)$ ). Let $R$ be a domain in $E$ and $m$ a positive integer. Then, a partition ${ }^{1}\left\{D_{1}, D_{2}, \cdots, D_{m}\right\}$ of $R \times\left(0, t_{0}\right)$ is said to be a regular partition of $R \times\left(0, t_{0}\right)$, provided for each $k(1 \leq k \leq m)$ there exists a piecewise smooth family of regions $R_{t}^{k}\left(0 \leq t \leq t_{0}\right)$ such that:

(i) $D_{k}$ is the open set in $E_{4}$ swept out by $R_{t}^{k}\left(0 \leq t \leq t_{0}\right)$.

(ii) If $(x, t) \in W_{k}^{*} \cap\left(R \times\left(0, t_{0}\right)\right)$, 娄 ${ }_{k}$ being the regular part of the surface $W_{k}$ swept out by $R_{t}^{k}\left(0 \leq t \leq t_{0}\right)$, then

$$
x^{k}(x, t) \neq 0 \text {, }
$$

where $x^{\mathrm{k}}$ is the normal component of the velocity of propagation of $\partial \mathrm{R}_{\mathrm{t}}^{\mathrm{k}}\left(0 \leq \mathrm{t} \leq \mathrm{t}_{\mathrm{o}}\right)$ as defined in $(2.4)$.

Observe that if $D_{1}$ is the open set in $E_{4}$ swept out by the piecewise smooth family of regions defined in (2.2), (2.1) and

$$
\mathrm{D}_{2}=\mathrm{R} \times(0,2 / \mathrm{c})-\overline{\mathrm{D}}_{1}, \mathrm{R}=\left\{\underset{\sim}{\mathrm{x}} \in \mathrm{E} \mid 0<\mathrm{x}_{\mathrm{i}}<1\right\},
$$

then $\left\{\mathrm{D}_{1}, \mathrm{D}_{2}\right\}$ is a regular partition of $\mathrm{R} \times(0,2 / \mathrm{c})$.

Condition (ii) is included in Definition 2.2, since we shall only by concerned here with propagating stress-discontinuities ${ }^{2}$. Note also that a regular partition of $R \times\left(0, t_{0}\right)$ defines, for each $t \in\left[0, t_{0}\right]$, a partition of $R$.

Definition 2. 3. (Piecewise continuous function of position and time, jumps). Let $R$ be a domain in $E$. A scalar function $f$ is said to be piecewise continuous on $\bar{R} \times\left[0, t_{0}\right]$, provided there exists a regular partition $\left\{\mathrm{D}_{1}, \mathrm{D}_{2}, \cdots, \mathrm{D}_{\mathrm{m}}\right\}$ of $\mathrm{R} \times\left(0, \mathrm{t}_{\mathrm{o}}\right)$ such that for every $\mathrm{k}(1 \leq \mathrm{k} \leq \mathrm{m})$,

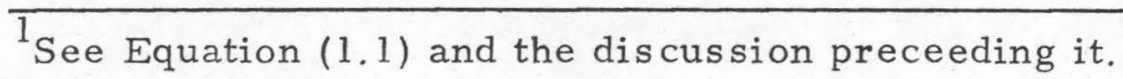

2 In fact, one can show that the traction vector, as well as the particle velocity, must be continuous across a stationary surface of stressdiscontinuity (see, for example, Keller [14]). 
$f$ is continuous on $D_{k}$ and coincides there with a function continuous on $\overline{\mathrm{D}}_{\mathrm{k}}$.

Further, letting W $_{k}$, for each $k(1 \leq k \leq m)$, be the regular part of the surface $W_{k}$ swept out by the family $R_{t}^{k}\left(0 \leq t \leq t_{o}\right)$ appropriate to $D_{k}$, we introduce the functions $(f)^{+},(f)^{-}$and $[f]$ on $\bigcup_{k=1}^{m} W_{k} \cap\left(R \times\left(0, t_{0}\right)\right)$ by means of

$$
\begin{aligned}
& (f)^{+}(\underset{\sim}{x}, t)=\lim _{h \rightarrow 0^{+}} f\left(\underset{\sim}{x}-h \hat{\sim}_{\sim}^{k}(\underset{\sim}{x}, t), t+h c k(\underset{\sim}{x}, t)\right), \\
& \text { (f) }{ }^{-}(\underset{\sim}{x}, t)=\lim _{h \rightarrow 0^{+}} f\left(\underset{\sim}{x+h \hat{\sim}^{k}}(\underset{\sim}{x}, t), t-h c{ }^{k}(\underset{\sim}{x}, t)\right), \\
& {[f](\underset{\sim}{x}, t)=(f)^{+}(\underset{\sim}{x}, t)-(f)^{-}(\underset{\sim}{x}, t)} \\
& \text { for all }(\underset{\sim}{\mathrm{x}}, \mathrm{t}) \in{\stackrel{*}{\mathrm{~W}_{\mathrm{k}}}}_{\mathrm{k}} \cap\left(\mathrm{R} \times\left(0, \mathrm{t}_{\mathrm{o}}\right)\right) \quad(\mathrm{k}=1, \cdots, \mathrm{m}) \text {. }
\end{aligned}
$$

Here, $\stackrel{\hat{\mathrm{n}}}{\sim}_{\sim}^{\mathrm{k}}$ and $\mathrm{c}^{\mathrm{k}}$ are the direction and the speed of propagation of the family $R_{t}^{k}\left(0 \leq t \leq t_{0}\right)$. The function $[f]$ on $W_{k}^{*} \cap\left(R \times\left(0, t_{0}\right)\right)$ is called the jump in $f$ across $W_{k} \cap\left(R \times\left(0, t_{0}\right)\right)$, and if $[f]$ does not vanish identically there, $W_{k} \cap\left(R \times\left(0, t_{0}\right)\right)$ is called a surface of discontinuity for $f$. Adopting analogous definitions for tensor fields of all orders, we are now in a position to formulate the traction problem for elastodynamic states with piecewise continuous stress fields. We require of these singular states that they satisfy the classical jump conditions, which are customarily derived from the balance of momentum postulate ${ }^{1}$. To arrive at a succinct statement of the singular boundary -

${ }^{1}$ See, for example, Truesdell and Toupin [2] (Section 205). It is assumed in [2] that the discontinuities occur across surfaces in $\mathrm{E}$ belonging to a smooth one-parameter family of smooth surfaces. We shall therefore require that the jump conditions hold on the regular parts of the surfaces carrying the discontinuities in stress. 
initial value problem to be considered, we introduce

Definition 2.4. (Piecewise regular elastodynamic state). Let $R$, $\left(0, t_{0}\right), \rho \underline{\text { and }} \underset{\sim}{\mathrm{c}}$ be as in Definition 1.1 and $\underset{\sim}{\mathrm{f}} \underset{\text { a vector field on }}{\mathrm{R}} \times\left[0, \mathrm{t}_{0}\right]$. We say that $S=[\underset{\sim}{u}, \sigma]$ is a piecewise regular elastodynamic state on $\overline{\mathrm{R}} \times\left[0, \mathrm{t}_{\mathrm{o}}\right]$, corresponding to the body-force field $\underset{\sim}{f}$, the mass-density field $\rho$ and the elasticity field $\underset{\sim}{\sim}$ provided:

(i) $\underset{\sim}{f} \in \mathbb{C}\left(\bar{R} \times\left[0, t_{0}\right]\right) ; \underset{\sim}{u} \in \mathbb{C}\left(\bar{R} \times\left[0, t_{0}\right]\right)$.

(ii) There exists a regular partition $\left\{D_{1}, D_{2}, \cdots, D_{m}\right\}$ of $R \times\left(0, t_{o}\right)$ such that for each $\mathrm{k}(1 \leq \mathrm{k} \leq \mathrm{m})$

$$
S \in \mathcal{E}\left(\underset{\sim}{f}, 0, \mathcal{c} ; D_{k}\right),
$$

and $S$ coincides on $D_{k}$ with a state $S^{k}=\left[\underset{\sim}{u^{k}}, \sigma_{\sim}^{k}\right]$ that is defined on $\bar{D}_{k}$ and satisfies

$$
\underset{\sim}{\sim}{ }^{k} \in c^{2}\left(\bar{D}_{k}\right), \quad \underset{\sim}{\sigma^{k}} \in c^{1}\left(\bar{D}_{k}\right)
$$

(iii) If $W_{k}$, for every $k(1 \leq k \leq m)$, is the surface swept out by the family $R_{t}^{k}\left(0 \leq t \leq t_{0}\right)$ appropriate to $D_{k}$, then $W_{k} \cap\left(R \times\left(0, t_{0}\right)\right)(k=1, \ldots, m)$ are surfaces of discontinuity for $\sigma$, and

$$
\hat{n}_{j}^{k}\left[\sigma_{i j}\right]=-\rho c^{k}\left[\dot{u}_{i}\right] \text { on } w_{k}^{*} \cap\left(R \times\left(0, t_{0}\right)\right) \quad(k=1, \ldots, m),
$$

where $\stackrel{\hat{n}}{\sim}_{\sim}^{k}$ and $c^{k}$ are the direction and the speed of propagation of the family $R_{t}^{k}\left(0 \leq t \leq t_{0}\right)$.

The surfaces $\mathrm{W}_{\mathrm{k}} \cap\left(\mathrm{R} \times\left(0, \mathrm{t}_{\mathrm{o}}\right)\right)(\mathrm{k}=1, \cdots, \mathrm{m})$ will henceforth be called the singular surfaces ${ }^{1}$ of $\mathrm{S}$. Also, we denote by

${ }^{1}$ Note that since each singular surface of $\mathrm{S}$ is a segment of the boundary of an element of a partition of $R \times\left(0, t_{0}\right)$, the singular surfaces of $\mathrm{S}$ cannot be pairwise disjoint. 


$$
\left.\varepsilon_{p} \underset{\sim}{(f, \rho, c} \underset{\sim}{-} ; \bar{R} \times\left[0, t_{0}\right]\right)
$$

the collection of all piecewise regular elastodynamic states on $\overline{\mathrm{R}} \times\left[0, \mathrm{t}_{\mathrm{o}}\right]$ corresponding to $\underset{\sim}{\mathrm{f}}, \rho$ and $\underset{\sim}{\sim}$.

Observe that if

$$
\mathrm{S}=[\underset{\sim}{\mathrm{u}} \underset{\sim}{\sigma}] \in \varepsilon_{\mathrm{p}}\left(\underset{\sim}{\sim}, \rho, \underset{\sim}{\mathrm{c}} ; \overline{\mathrm{R}} \times\left[0, \mathrm{t}_{0}\right]\right),
$$

$\underset{\sim}{\sigma}$ is defined merely on $\overline{\mathrm{R}} \times\left[0, \mathrm{t}_{0}\right]-\overline{\mathrm{A}}$, where

$$
A=\bigcup_{k=1} W_{k} \cap\left(R \times\left(0, t_{o}\right)\right) \text {. }
$$

Thus, if $R$ is a regular region, the traction vector $\underset{\sim}{S}$ of $S$ on $\partial R$ is given by

$$
s_{i}=n_{j} \sigma_{i j} \text { on } \stackrel{*}{\partial} R \times\left[0, t_{0}\right]-\bar{A} \text {. }
$$

We remark further that the jump conditions (2.8) in Definition 2.4 may be replaced by

$$
\begin{aligned}
& a_{i j}^{k}\left[\dot{u}_{j}\right]=\left(c^{k}\right)^{2}\left[\dot{u}_{i}\right] \text { on } \stackrel{*}{W}_{k}^{*} \cap\left(R \times\left(0, t_{0}\right)\right) \quad(k=1, \cdots, m), \\
& a_{i j}^{k}=\frac{1}{\rho} c_{i p j q} \hat{n}_{p}^{k} \hat{n}_{q}^{k} \text { on } \stackrel{*}{W}_{k}^{*} \cap\left(R \times\left(0, t_{o}\right)\right) \quad(k=1, \cdots, m),
\end{aligned}
$$

there being no sum on $\mathrm{k}$ in (2.12). The equivalence of (2.11) and (2.8) can be established by reversing the steps in the familiar argument ${ }^{1}$ for deducing (2.11) from (2.8). From (2.11) it follows in particular that the propagation speeds $c^{k}(k=1, \cdots, m)$ in Definition 2.4 must be wave speeds for the material with the elasticity field $\underset{\sim}{c}$.

The analogue of the classical traction problem in the present context is the traction problem for piecewise regular elastodynamic states, which may be stated as follows. Given a regular region $\mathrm{R}$ and

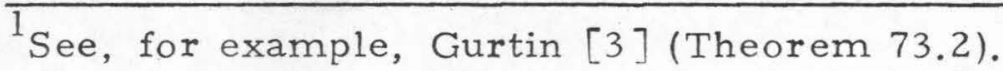


a time-interval $\left(0, t_{0}\right)$, find

$$
S=[\underset{\sim}{u}, \sigma] \in \varepsilon_{p}\left(\underset{\sim}{\sim}, \rho, c ; \bar{R} \times\left[0, t_{0}\right]\right)
$$

for the data $\underset{\sim}{f}, \rho, \underset{\sim}{c}$ and subject to the initial conditions

$$
\underset{\sim}{\mathrm{u}}(\cdot, 0)=\stackrel{\stackrel{\mathrm{u}}{\sim}}{\sim} \text { on } \overline{\mathrm{R}} \quad, \quad \underset{\sim}{\mathrm{u}}(\cdot, 0)=\stackrel{\circ}{\mathrm{V}} \text { on } \overline{\mathrm{R}}-\overline{\mathrm{A}}
$$

and the boundary condition

$$
\underset{\sim}{\mathrm{s}}=\stackrel{*}{\underset{\sim}{\mathrm{S}}} \text { on } \stackrel{*}{\partial} \mathrm{R} \times\left[0, \mathrm{t}_{\mathrm{o}}\right]-\overline{\mathrm{A}}
$$

where $\stackrel{\stackrel{\sim}{u}}{\sim}, \underset{\sim}{\stackrel{\sim}{\sim}}$ and $\underset{\sim}{\stackrel{*}{\sim}}$ are prescribed functions and $A$ is the union of all the singular surfaces of $S$ (see (2.9)).

In preparation for a uniqueness theorem appropriate to the problem just formulated, we derive from Equations (2.8) another set of jump relations which will play a crucial role in the proof of an energy identity for piecewise regular elastodynamic states.

Theorem 2.1. (Energy balance across singular surfaces). If

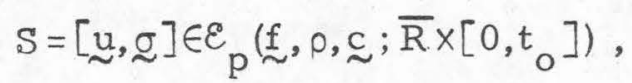

and $\underset{\sim}{\hat{n}^{k}}, c^{k}$ and $\stackrel{*}{W}_{k}$ are as in Definition 2.4 , then

$$
\hat{n}_{j}^{k}\left[\sigma_{i j} \dot{u}_{i}\right]+\frac{c^{k}}{2}\left(\rho\left[\dot{u}_{j} \dot{u}_{j}\right]+\left[\sigma_{i j} u_{i, j}\right]\right)=0 \text { on } \stackrel{*}{*}_{k} \cap\left(R \times\left(0, t_{o}\right)\right) \quad(k=1, \cdots, m) \text {. }
$$

Proof: Fix $\mathrm{k}(1 \leq \mathrm{k} \leq \mathrm{m})$ and put

$$
e=\hat{n}_{j}^{k}\left[\sigma_{i j} \dot{u}_{i}\right]+\frac{c^{k}}{2}\left(\rho\left[\dot{u}_{j} \dot{u}_{j}\right]+\left[\sigma_{i j} u_{i, j}\right]\right) \text { on } \stackrel{*}{*}_{k}^{k} \cap\left(R \times\left(0, t_{o}\right)\right) \text {. }
$$

Next, use (2.7) repeatedly to see that the following relations hold on $\stackrel{*}{W}_{\mathrm{k}} \cap\left(\mathrm{R} \times\left(0, \mathrm{t}_{\mathrm{o}}\right)\right)$ : 


$$
\begin{gathered}
{\left[\sigma_{i j} \dot{u}_{i}\right]=\left(\dot{u}_{i}\right)^{+}\left[\sigma_{i j}\right]+\left(\sigma_{i j}\right)^{-}\left[\dot{u}_{i}\right] ;} \\
{\left[\dot{u}_{j} \dot{u}_{j}\right]=\left[\dot{u}_{j}\right]\left[\dot{u}_{j}\right]+2\left(\dot{u}_{j}\right)^{-}\left[\dot{u}_{j}\right] ;} \\
{\left[\sigma_{i j} u_{i, j}\right]=\left[\sigma_{i j}\right]\left[u_{i, j}\right]+\left(u_{i, j}\right)^{-}\left[\sigma_{i j}\right]+\left(\sigma_{i j}\right)^{-}\left[u_{i, j}\right] .}
\end{gathered}
$$

But, according to a well-known theorem usually attributed to Maxwell ${ }^{1}$,

$$
\hat{n}_{j}^{k}\left[\dot{u}_{i}\right]=-c k\left[u_{i, j}\right] \text { on } \stackrel{*}{W}_{k} \cap\left(R \times\left(0, t_{0}\right)\right)
$$

for any vector field $\underset{\sim}{u}$ satisfying the regularity assumption in (ii) of Definition 2.4. Hence, by (B), (E) and the jump conditions (2.8),

$$
\hat{n}_{j}^{k}\left[\sigma_{i j} \dot{u}_{i}\right]=-\rho c^{k}\left(\dot{u}_{i}\right)^{+}\left[\dot{u}_{i}\right]-c^{k}\left(\sigma_{i j}\right)^{-}\left[u_{i, j}\right] \text { on } W_{k}^{*} \cap\left(R \times\left(0, t_{o}\right)\right) \text {. }
$$

Substitution from (C), (D) and (F) into (A) results in

$$
\frac{2 e}{c^{k}}=\left[\sigma_{i j}\right]\left[u_{i, j}\right]-\rho\left[\dot{u}_{j}\right]\left[\dot{u}_{j}\right]+\left(u_{i, j}\right)^{-}\left[\sigma_{i j}\right]-\left(\sigma_{i j}\right)^{-}\left[u_{i, j}\right] \text { on } w_{k}^{*} \cap\left(R \times\left(0, t_{o}\right)\right) \text {. }
$$

On the other hand, using (E) and then (2.8), we obtain

$$
\left[\sigma_{i j}\right]\left[u_{i, j}\right]=\rho\left[\dot{u}_{j}\right]\left[\dot{u}_{j}\right] \text { on } w_{k}^{*} \cap\left(R \times\left(0, t_{0}\right)\right) \text {. }
$$

Also, the symmetry-relations (1.5) for $\underset{\sim}{ }$ the stress-displacement relations (1.6) and the postulated smoothness of $\underset{\sim}{u}$ and $\underset{\sim}{\sigma}$ allow us to conclude that

$$
\left(\sigma_{i j}\right)^{-}\left[u_{i, j}\right]=\left(u_{i, j}\right)^{-}\left[\sigma_{i j}\right] \text { on } W_{k}^{*} \cap\left(R \times\left(0, t_{o}\right)\right) \text {. }
$$

Upon inserting $(\mathrm{H})$ and $(\mathrm{I})$ into $(\mathrm{G})$ and using $(\mathrm{A})$, we arrive at (2.15). The theorem is thus proved.

For a proof, see, for instance, Gurtin [3] (Theorem 72.1). 
The four-dimensional divergence theorem for vector fields defined on subsets of $\mathrm{E}_{4}$ swept out by piecewise smooth families of regions is the burden of

Lemma 2.1. Let $R_{t}\left(t_{1} \leq t \leq t_{2}\right)$ be a piecewise smooth family of regions sweeping out $\mathrm{D} \subset \mathrm{E}_{4} . \quad$ Suppose also that $\underset{\sim}{\mathrm{p}}$ and $\mathrm{q}$ are, respectively, $\underline{\mathrm{a}}$ vector and scalar field on $\bar{D}$ satisfying

$$
\underset{\sim}{p} \in C^{1}(\bar{D}) \quad, \quad q \in C^{1}(\bar{D}) \text {. }
$$

Then,

$$
\begin{aligned}
& \int^{t_{2}} \int(\nabla \cdot \underset{\sim}{p}+\dot{q}) d V d t=\int^{t_{2}} \int(\underset{\sim}{n} \cdot \underset{\sim}{p}-\mu q) d A d t+\int q\left(\underset{\sim}{x}, t_{2}\right) d V-\int q\left(\underset{\sim}{x}, t_{1}\right) d V, \\
& { }_{t_{1} R_{t}}^{t_{1} \partial R_{t}} \quad \mathrm{R}_{t_{2}} \quad R_{t_{1}}
\end{aligned}
$$

where $\underset{\sim}{\mathrm{n}}(\cdot, \mathrm{t})$ and $u(\cdot, \mathrm{t})$, for every $\mathrm{t}\left(\mathrm{t}_{1} \leq \mathrm{t} \leq \mathrm{t}_{2}\right)$, are the unit outward normal and the normal component of the velocity of propagation of $\partial R_{t}$.

Proof: Let $\left\{\tau_{0}, \tau_{1}, \cdots, \tau_{m}\right\}$ be the partition of $\left(t_{1}, t_{2}\right)$ accompanying $R_{t}\left(t_{1} \leq t \leq t_{2}\right)$ in Definition 2.1. Next, fix $k(1 \leq k \leq m)$ and let $\varphi$ be the mapping function for the smooth family of regions $R_{t}\left(\tau_{k-1} \leq t \leq \tau_{k}\right)$.

Then, according to Definition 2.1 and the smoothness hypothesis on $\underset{\sim}{\mathrm{p}}$, we may conclude from the three-dimensional divergence theorem that

$$
\left.\left.\int_{\mathrm{R}_{\mathrm{t}}} \nabla \cdot \underset{\sim}{\mathrm{p}} \underset{\sim}{\mathrm{x}}, \mathrm{t}\right) \mathrm{dV}=\underset{\partial \mathrm{R}_{\mathrm{t}}}{\int_{\sim}^{\mathrm{n}}} \underset{\sim}{\mathrm{x}}, \mathrm{t}\right) \cdot \underset{\sim}{\mathrm{p}}(\underset{\sim}{\mathrm{x}}, \mathrm{t}) \mathrm{dA} \text { for every } \mathrm{t} \in\left(\tau_{\mathrm{k}-1}, \tau_{\mathrm{k}}\right) .
$$

Moreover, setting 


$$
\left.J(\underset{\sim}{x}, t)=\left.\frac{\partial\left(\varphi_{1}, \varphi_{2}, \varphi_{3}\right)}{\partial\left(x_{1}, x_{2}, x_{3}\right)}\right|_{(\underset{\sim}{x}, t)} \text { for every } \underset{\sim}{x}, t\right) \in \bar{R}_{\hat{t}} \times\left[\tau_{k-1}, \tau, k\right],
$$

we have

$$
\left.\left.\int_{\mathrm{R}_{\mathrm{t}}} \nabla \cdot \underset{\sim}{\mathrm{p}} \underset{\sim}{\mathrm{x}}, \mathrm{t}\right) \mathrm{dV}=\int_{\mathrm{R}_{\hat{\mathrm{t}}}} \nabla \cdot \underset{\sim}{\mathrm{p}}(\underset{\sim}{\varphi} \underset{\sim}{\mathrm{x}}, \mathrm{t}), \mathrm{t}\right) \mathrm{J}(\underset{\sim}{\mathrm{x}}, \mathrm{t}) \mathrm{dV} \text { for each } \mathrm{t} \in\left(\tau_{\mathrm{k}-1}, \tau_{\mathrm{k}}\right) .
$$

From (C), the smoothness of $\varrho$ asserted in Definition 2.1, and the assumed regularity of $\mathrm{p}$, we see that both members of $(A)$ can be integrated from $\tau_{k-1}$ to $\tau_{k}$. Thus,

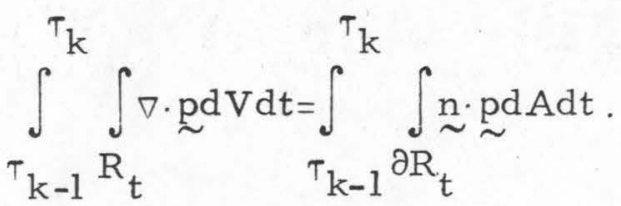

Further,

$$
\int_{R_{t}} q(\underset{\sim}{x}, t) d V=\int_{R_{\hat{t}}} q(\underset{\sim}{\varphi}(\underset{\sim}{x}, t), t) J(\underset{\sim}{x}, t) d V \text { for all } t \in\left(\tau_{k-1}, \tau_{k}\right),
$$

so that, taking advantage of the smoothness of $\mathrm{q}$ and $\varphi$ and the familiar identity $^{1}$

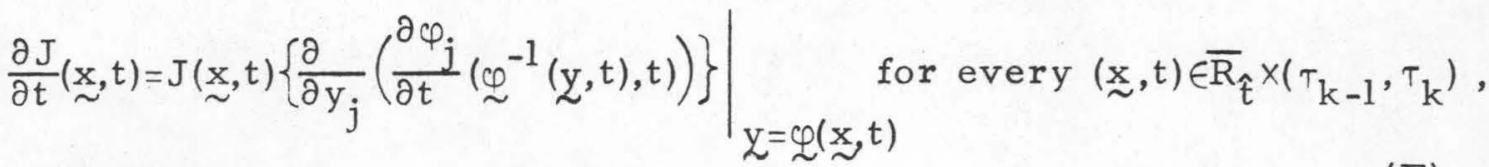

we infer

${ }^{1}$ Cf. , Truesdell and Toupin [2] (Section 76). Observe that the required smoothness of $\varphi^{-1}$ follows from the inverse function theorem. See, for example, Buck [15] (p. 278). 


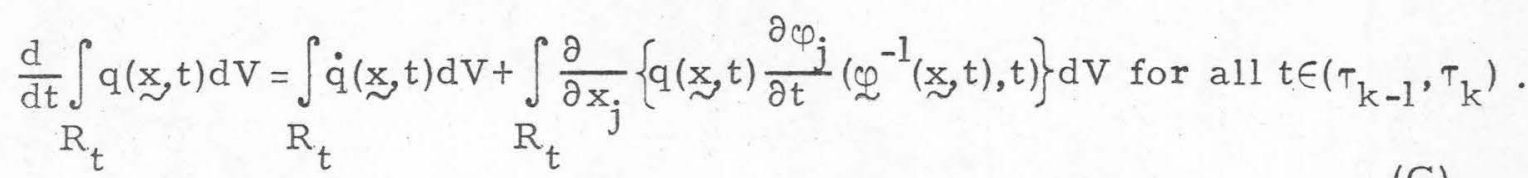

Next, apply the three-dimensional divergence theorem to the second integral on the right side of $(G)$, to arrive at

$$
\left.\underset{R_{t}}{\frac{d}{d t}} \int_{\sim} q(\underset{\sim}{x}, t) d V=\int_{R_{t}} \dot{q}(\underset{\sim}{x}, t) d V+\int_{\partial R_{t}}^{q} \underset{\sim}{x}, t\right) u(\underset{\sim}{x}, t) d A \text { for all } t \in\left(\tau_{k-1}, \tau_{k}\right),
$$

where $x$ is given by (2.4). It is an easy matter to justify the integration of both sides of $(\mathrm{H})$ from $\tau_{k-1}$ to $\tau_{k}$. We thus have,

$$
\int_{\tau_{k-1}}^{\tau_{k}} \int_{t} q d V d t+\int_{\tau_{k-1}}^{\tau_{k}} \int_{\partial R_{t}} x q d A d t=\lim _{t \rightarrow \tau_{k}^{-}} \int_{R_{t}} q(\underset{\sim}{x}, t) d V-\lim _{t \rightarrow \tau_{k-1}^{+}} \int_{R_{t}} q(\underset{\sim}{x}, t) d V .
$$

Now (I) holds for every $\mathrm{k}(1 \leq \mathrm{k} \leq \mathrm{m})$. Moreover, as is easily verified,

$$
\lim _{t \rightarrow T_{k}} \int_{R_{t}} q(x, t) d V=\int_{R_{k}} q\left(x, T_{k}\right) d V \quad(k=0,1, \cdots, m) .
$$

Thus, summing (D) and (I) over $k(1 \leq k \leq m)$ and adding the resulting equations yields (2.16). This completes the proof.

Lemma 2.1 coupled with Theorem 2.1 enables us to establish Theorem 2.2. (Energy identity for piecewise regular elastodynamic states). Let $\mathrm{R}$ be a regular region and

$$
S=[\underset{\sim}{\sim}, \sigma] \in \mathcal{E}_{\mathrm{p}}\left(\underset{\sim}{f}, \rho, \underset{\sim}{\sim} ; \overline{\mathrm{R}} \times\left[0, \mathrm{t}_{0}\right]\right) .
$$

Then, for every $t \in\left[0, t_{0}\right]$, one has 


$$
\begin{aligned}
& \iint \underset{\sim}{t} \underset{\sim}{\dot{u}} d V d \tau+\int^{t} \int \underset{\sim}{s} \cdot \underset{\sim}{\dot{u}} d A d \tau=E(t)-E(0), \\
& \begin{array}{ll}
0 \mathrm{R} & 0 \partial \mathrm{R}
\end{array}
\end{aligned}
$$

where

$$
E(t)=\frac{1}{2} \int_{R} \rho(\underset{\sim}{x}) \underset{\sim}{\dot{u}^{2}}(\underset{\sim}{x}, t) d V+\frac{1}{2} \int_{R} \underset{\sim}{\sigma}(x, t) \cdot \underset{\sim}{\sim} \underset{\sim}{u}(x, t) d V .
$$

Proof: Let $\left\{D_{1}, D_{2}, \ldots, D_{m}\right\}$ be the regular partition of $R \times\left(0, t_{0}\right)$ appropriate to $\mathrm{S}$ in the sense of Definition 2.4 and fix $\mathrm{k}(1 \leq \mathrm{k} \leq \mathrm{m})$.

Next, define a vector field $\underset{\sim}{\mathrm{p}}$ on $\overline{\mathrm{D}}_{\mathrm{k}}$ by setting

$$
p_{j}=-\sigma_{i j} \dot{u}_{i} \text { in } D_{k}, \underset{\sim}{p} \in c^{l}\left(\bar{D}_{k}\right) .
$$

That this is possible is readily seen from (ii) of Definition 2.4. Similarly, define a scalar field $\mathrm{q}$ on $\overline{\mathrm{D}}_{\mathrm{k}}$ through

$$
q=\frac{1}{2} \rho \dot{u}_{i} \dot{u}_{i}+\frac{1}{2} \sigma_{i j} u_{i, j} \text { in } D_{k}, q \in C^{1}\left(\bar{D}_{k}\right) \text {. }
$$

An elementary computation involving a use of the stress equations of motion (1.7), the stress-displacement relations (1.6) and the symmetry of $\underset{\sim}{\mathrm{c}}$ asserted in (1.5) yields

$$
p_{j, j}+\dot{q}=f_{j} \dot{u}_{j} \text { in } D_{k} \text {. }
$$

By hypothesis and Definitions 2.4,2.2, $\mathrm{D}_{\mathrm{k}}$ is swept out by a piecewise smooth family of regions $R_{\tau}^{k}\left(0 \leq \tau \leq t_{0}\right)$. Moreover, it follows from Definition 2.1 that, for any fixed $t \in\left(0, t_{0}\right)$, the oneparameter family of sets $R_{\tau}^{\mathrm{k}}(0 \leq \tau \leq t)$ is also a piecewise smooth family of regions. Thus, we conclude from Lemma 2.1 applied to $\underset{\sim}{\mathrm{p}}, \mathrm{q}$ on the region in $E_{4}$ swept out by $R_{\tau}^{k}(0 \leq \tau \leq t)$ that 


$$
\begin{aligned}
& \left.\left.\int_{0}^{t} \int_{R_{T}^{k}} f_{j} \underset{\sim}{(x}, \tau\right) \dot{u}_{j} \underset{\sim}{(x}, \tau\right) d V d \tau=\int_{0}^{t} \int_{\partial R_{\tau}^{k}}\left(n_{j}^{k} \underset{\sim}{(x, \tau) p_{j}}(\underset{\sim}{x}, \tau)-x^{k}(\underset{\sim}{x}, \tau) q(\underset{\sim}{x}, \tau)\right) d V d \tau \\
& +\frac{1}{2} \int_{R_{t}^{k}}\left(\rho(\underset{\sim}{x}) \dot{u}_{j}(\underset{\sim}{x}, t) \dot{u}_{j}(\underset{\sim}{x}, t)+\sigma_{i j}(\underset{\sim}{\sim}, t) u_{i, j}(\underset{\sim}{x}, t)\right) d V
\end{aligned}
$$

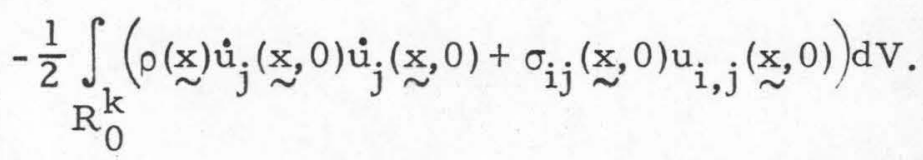

Observe that, according to $(A)$ and $(B), \underset{\sim}{\mathrm{p}}$ and $\mathrm{q}$ are defined on $\mathrm{W}_{\mathrm{k}}^{*}-$ the regular part of the surface $W_{k}$ swept out by $R_{\tau}^{k}(0 \leq \tau \leq t)-$ through their limits as $\stackrel{*}{W}_{k}$ is approached from within $D_{k}$. Also, if $\stackrel{*}{W}_{k} \cap\left(\partial R \times\left[0, t_{0}\right]\right)$ is not empty,

$$
x^{\mathrm{k}}=0 \quad, \quad \mathrm{n}^{\mathrm{k}}=\underset{\sim}{\mathrm{n}} \text { on } \stackrel{*}{\mathrm{~W}}_{\mathrm{k}} \cap\left(\partial \mathrm{R} \times\left[0, \mathrm{t}_{\mathrm{o}}\right]\right),
$$

where $\underset{\sim}{\mathrm{n}}$ is the normal of $\partial \mathrm{R}$. Thus, the integrand of the boundary integral in (D) is given by

$$
\mathrm{n}_{j}^{\mathrm{k}} \mathrm{p}_{j}-\varkappa^{\mathrm{k}} \mathrm{q}=-\mathrm{n}_{j} \sigma_{i j} \dot{\mathrm{u}}_{\mathrm{i}} \quad \text { on } \quad \stackrel{*}{W}_{\mathrm{k}} \cap\left(\partial \mathrm{R} \times\left[0, \mathrm{t}_{0}\right]\right)
$$

provided $\stackrel{*}{W}_{k} \cap\left(\partial R \times\left[0, t_{0}\right]\right)$ is non-void, and

$n_{j}^{k} p_{j}-x^{k} q=e_{k}^{+} \equiv-n_{j}^{k}\left(\sigma_{i j} \dot{u}_{i}\right)^{+}-\frac{x^{k}}{2}\left\{\rho\left(\dot{u}_{j} \dot{u}_{j}\right)^{+}+\left(\sigma_{i j} u_{i, j}\right)^{+}\right\} \quad$ on $\quad \stackrel{*}{w}_{k}^{*} \cap\left(R \times\left(0, t_{o}\right)\right)$,

or

$n_{j}^{k} p_{j}-x^{k} q=e_{k}^{-} \equiv-n_{j}^{k}\left(\sigma_{i j} \dot{u}_{i}\right)^{-}-\frac{x^{k}}{2}\left\{\rho\left(\dot{u}_{j} \dot{u}_{j}\right)^{-}+\left(\sigma_{i j} u_{i, j}\right)^{-}\right\}$on $\stackrel{*}{w}_{k}^{*} \cap\left(R \times\left(0, t_{0}\right)\right)$,

according as $x^{\mathrm{k}}$ is positive or negative. On the other hand, from

Theorem 2.1 in conjunction with (2.5), (2.6) and (2.7), we see that $\mathrm{e}_{\mathrm{k}}^{+}$ and $e_{k}^{-}$coincide on $W_{k}^{*} \cap\left(R \times\left(0, t_{o}\right)\right)$. Hence, in view of $(E),(F)$ and $(G)$, 
we may rewrite (D) in the form

$$
\begin{aligned}
& \int_{0}^{t} \int_{R_{T}^{k}} f_{j} \dot{u}_{j} d V d \tau+\int_{0}^{t} \int_{\partial R_{\tau}^{k} \cap \partial R} n_{j} \sigma_{i j} \dot{u}_{i} d A d \tau=\int_{0}^{t} \int_{\partial R^{k}{ }_{\tau}} e_{k} d A d \tau \\
& +\int_{R_{t}^{k}} \frac{1}{2}\left(\rho \dot{u}_{j} \dot{u}_{j}+\sigma_{i j} u_{i, j}\right) d V-\int_{R_{0}^{k}} \frac{1}{2}\left(\rho \dot{u}_{j} \dot{u}_{j}+\sigma_{i j} u_{i, j}\right) d V,
\end{aligned}
$$

where the arguments of the integrands in $(\mathrm{H})$ are the same as in (D), and $e_{k}$ is the common value of $e_{k}^{+}$and $e_{k}^{-}$on $\stackrel{W}{*}_{k}^{*} \cap\left(R \times\left(0, t_{0}\right)\right)$.

Finally, note that $(\mathrm{H})$ is true for every $\mathrm{k}(1 \leq \mathrm{k} \leq \mathrm{m})$. Moreover, $\left\{\stackrel{\circ}{R}_{\tau}^{1}, \circ_{R}^{2}, \ldots, \stackrel{\circ}{R}_{\tau}^{m}\right\}$, for each $\tau \in\left[0, t_{0}\right]$, partitions $R$. Therefore, if we sum $(\mathrm{H})$ over $\mathrm{k}(1 \leq \mathrm{k} \leq \mathrm{m})$, the boundary integrals from the right side cancel, and we reach the desired result (2.17).

Just as the classical energy identity (1.11) leads to Neumann's uniqueness theorem (Theorem 1.2), so (2.17) gives rise to Theorem 2.3. (Uniqueness of solution to the traction problem for piecewise regular elastodynamic states). Let $\mathrm{R}$ be a regular region and

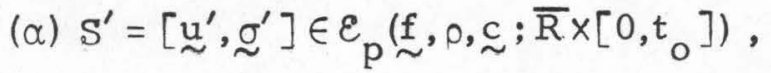

$$
\begin{aligned}
& S^{\prime \prime}=\left[{\underset{\sim}{u}}^{\prime \prime}, \sigma^{\prime \prime}\right] \in \varepsilon_{\mathrm{p}}\left(\underset{\sim}{f}, \rho, \mathrm{c} ; \overline{\mathrm{R}} \times\left[0, \mathrm{t}_{\mathrm{o}}\right]\right),
\end{aligned}
$$

with respective singular surfaces

$$
\mathrm{W}_{\mathrm{k}}^{\prime} \cap\left(\mathrm{R} \times\left(0, \mathrm{t}_{\mathrm{o}}\right)\right) \quad\left(\mathrm{k}=\mathrm{l}, \cdots, \mathrm{m}^{\prime}\right) \quad, \quad \mathrm{W}_{\mathrm{k}}^{\prime \prime} \cap\left(\mathrm{R} \times\left(0, \mathrm{t}_{\mathrm{o}}\right)\right) \quad\left(\mathrm{k}=1, \cdots, \mathrm{m}^{\prime \prime}\right) .
$$

Suppose also that $S=[\underset{\sim}{\sim}, \underset{\sim}{\sigma}]$, defined by

$$
\underset{\sim}{\mathrm{u}}=\underline{\sim}^{\prime}-\underline{\sim}^{\prime \prime} \text { on } \overline{\mathrm{R}} \times\left[0, \mathrm{t}_{\mathrm{o}}\right], \quad \underset{\sim}{\sim} \sigma^{\prime}-\sigma^{\prime \prime} \text { on } \overline{\mathrm{R}} \times\left[0, \mathrm{t}_{\mathrm{o}}\right]-\overline{\mathrm{A}}
$$


where

$$
A=\left\{\bigcup_{k=1}^{m^{\prime}} W_{k}^{\prime} \cap\left(R \times\left(0, t_{o}\right)\right)\right\} \cup\left\{\bigcup_{k=1}^{m^{\prime \prime}} W_{k}^{\prime \prime} \cap\left(R \times\left(0, t_{o}\right)\right)\right\},
$$

$\underline{\text { satisfies: }}$

$$
\begin{aligned}
& \text { (ß) } s \in \varepsilon_{\mathrm{p}}\left(\underset{\sim}{0}, p, \underset{\sim}{\mathrm{c}} ; \overline{\mathrm{R}} \times\left[0, \mathrm{t}_{\mathrm{o}}\right]\right) \text {. } \\
& \text { (Y) } \underset{\sim}{\mathrm{u}}(\cdot, 0)=\underset{\sim}{0} \text { on } \overline{\mathrm{R}}, \quad \underset{\sim}{\mathrm{u}}(\cdot, 0)=\underset{\sim}{0} \text { on } \overline{\mathrm{R}}-\overline{\mathrm{A}} \text {, } \\
& \mathrm{s}=0 \text { on }{ }^{\text {社 }} \mathrm{R} \times\left[0, \mathrm{t}_{\mathrm{O}}\right]-\overline{\mathrm{A}} \text {. }
\end{aligned}
$$

In addition, as sume

( $\delta) \cong$ is positive semi-definite on $R$, so that

$$
c_{i j k \ell} \stackrel{(x)}{\sim} Y_{i j} Y_{k \ell} \geq 0 \text { for } \underset{\text { all }}{\underset{\sim}{x} \in R}
$$

and for every symmetric two-tensor $\mathcal{Y}$. Then,

$$
\stackrel{u}{\sim}^{\prime}=\sim^{\prime \prime} \text { on } \bar{R} \times\left[0, t_{0}\right], \quad \sigma^{\prime}=\sigma^{\prime \prime} \text { on } \bar{R} \times\left[0, t_{0}\right]-\bar{A} .
$$

The purpose of Assumption $(\beta)$ in the statement of Theorem 2.3 is to insure that the subsets of $\mathrm{R} \times\left(0, \mathrm{t}_{\mathrm{O}}\right)$, on which the difference-state $S$ is smooth, constitute a regular partition of $R \times\left(0, t_{0}\right)$. This fact, in turn, permits an application of the energy identity (2.17) to S. Note that $(\beta)$ is implied by Hypothesis $(\alpha)$ if the partitions appropriate to $S^{\prime}$ and $S^{\prime \prime}$ coincide, so that in this instance $(\beta)$ may be omitted.

Proof of Theorem 2.3: From $(\beta),(\gamma)$ and the energy identity (2.17) applied to $\mathrm{S}$, one draws

$$
\left.\int_{\mathrm{R}} \rho(\underset{\sim}{\mathrm{x}}) \dot{\mathrm{u}}_{\sim}^{2}(\underset{\sim}{\mathrm{x}}, \mathrm{t}) \mathrm{dV}+\int_{\mathrm{R}} \underset{\sim}{\sigma(\mathrm{x}}, \mathrm{t}\right) \cdot \underset{\sim}{\nabla} \underset{\sim}{\mathrm{u}}(\mathrm{t}) \mathrm{dV}=0 \text { for all } \mathrm{t} \in\left[0, \mathrm{t}_{\mathrm{o}}\right] .
$$


But according to Assumption ( $\delta$ ) and the stress-displacement relations (1.6), the second integral in (A) is non-negative. Therefore, (A) implies

$$
\int_{\mathrm{R}} \rho(\underset{\sim}{\mathrm{x}}) \dot{\sim}^{2}(\underset{\sim}{\mathrm{x}}, \mathrm{t}) \mathrm{dV}=0 \text { for all } \mathrm{t} \in\left[0, \mathrm{t}_{\mathrm{o}}\right] \text {, }
$$

which, in view of the piecewise continuity of $\underset{\sim}{\dot{\sim}}$ and the positivity of $\rho$, gives

$$
\underset{\sim}{\dot{u}}=0 \text { on } \bar{R} \times\left[0, t_{0}\right]-\bar{A} \text {. }
$$

Because of the continuity of $\underset{\sim}{u}$ on $\bar{R} \times\left[0, t_{0}\right]$ and the first initial condition in $(Y)$, the identity (C) yields

$$
\underset{\sim}{\mathrm{u}}=\underset{\sim}{0} \text { on } \overline{\mathrm{R}} \times\left[0, \mathrm{t}_{\mathrm{o}}\right]
$$

Finally, (D), together with the stress-displacement relations (1.6), implies

$$
\underset{\sim}{\sigma=0} \sim \text { on } \overline{\mathrm{R}} \times\left[0, \mathrm{t}_{0}\right]-\overline{\mathrm{A}}
$$

The proof is now complete.

As will become apparent at the end of the next section, the conclusion in Theorem 2.3 remains valid even if the somewhat artificial Assumption $(\beta)$ is deleted, provided the region $\mathrm{R}$ is restricted in a slightly different manner. Indeed, this result will emerge as a special case of a more inclusive uniqueness theorem given in Section 3 , where we consider the traction problem for states that may be considerably more singular than those treated here. Lastly, we observe that there is no difficulty in using the energy identity (2.17) to establish a generalization of Theorem 2.3 to the corresponding mixed boundary- 
initial value problem, which contains the first and second problems as special cases. 
3. Uniqueness of solution to the traction problem for weak elastodynamic states.

Our present objective is to relax the previous regularity assumptions on solutions to the second boundary-initial value problem of elastodynamics and to deal with the broader uniqueness issue thus arising. Following certain required preliminaries, we introduce the notion of a "weak elastodynamic state" and subsequently formulate the traction problem for such a state. We then prove an appropriate uniqueness theorem by extending an argument used by Ladyzhenskaya [5] in connection with the first boundary-initial value problem for a second-order hyperbolic equation in a single dependent variable.

Throughout this section we use the symbol $\mathcal{L}_{2}(D)$ for the class of all tensor-valued functions that are (Lebesgue) square-summable ${ }^{1}$ on a bounded region $\mathrm{D}$ in $\mathrm{E}_{\mathrm{n}}$. Furthermore, suppose $\mathrm{I}$ is a timeinterval and $S$ a regular surface element ${ }^{2}$ in $E$, so that for some choice of a Cartesian frame in $\mathrm{E}, \mathrm{S}$ admits the representation

$$
x_{3}=G\left(x_{1}, x_{2}\right) \text { for all }\left(x_{1}, x_{2}\right) \in \Pi, \quad G \in C^{1}(\Pi) \text {, }
$$

where $\Pi$ is a closed region in the $\left(x_{1}, x_{2}\right)$-plane. We say that a function is measurable on $S \times I$ if the corresponding composite function on $\Pi \times I$ has this property. We are thus in a position to speak of functions measurable on $\partial R \times I$ whenever $R$ is a regular region of space.

\footnotetext{
${ }^{1}$ As is customary, we call a measurable function summable if its Lebesgue integral is finite and square-summable if its square is summable.

${ }^{2}$ See Kellogg [117, p. 105.
} 
If $D$ is a bounded domain in $E_{n}$, we denote by $\hat{C}^{n}(\bar{D})$ the subclass of $C^{n}(\bar{D})$ consisting of all vector fields the supports ${ }^{1}$ of which are contained in D. Consequently, a function $\underset{\sim}{\varphi} \in \hat{C}^{n}(\bar{D})$ vanishes identically in a neighborhood of $\partial \mathrm{D}$. Next, suppose $\mathrm{R}$ is a bounded domain in $\mathrm{E}$ and $\underset{\sim}{p}$ a vector field, summable on $\bar{R} \times\left[0, t_{0}\right]$. If there exists a vector field $\underset{\sim}{\mathrm{v}}$ and a two-tensor field $\underset{\sim}{\mathrm{w}}$, both summable on $\overline{\mathrm{R}} \times\left[0, \mathrm{t}_{0}\right]$, such that for every $\varphi \in \hat{C}^{l}\left(\bar{R} \times\left[0, t_{0}\right]\right)$

$$
\int_{0}^{t_{0}} \int_{R_{i}} \dot{\varphi}_{i} p_{i} d v d t=-\int_{0}^{t_{0}} \int_{R} \varphi_{i} v_{i} d v d t,
$$

$$
\int_{0}^{t_{0}} \int_{R_{i}} \varphi_{i} p_{i} d V d t=-\int_{0}^{t_{0}} \int_{R^{\prime}} \varphi_{i j} w_{i j} d V d t,
$$

we say that $\underset{\sim}{\mathrm{p}}$ possesses a generalized time-derivative $\underset{\sim}{\mathrm{v}}$ and a generalized gradient $\underset{\sim}{\mathrm{w}}$ on $\overline{\mathrm{R}} \times\left[0, \mathrm{t}_{\mathrm{o}}\right]$. If $\underset{\sim}{\mathrm{v}}$ and $\underset{\sim}{\mathrm{v}^{\prime}}$ are both generalized timederivatives of $\underset{\sim}{p}$ on $\bar{R} \times\left[0, t_{0}\right]$, then (3.1) implies

$$
\begin{aligned}
& \text { to } \\
& \iint \varphi \cdot\left(\underset{\sim}{v}-v_{\sim}^{\prime}\right) d V d t=0 \text { for every } \varphi \in \hat{C}^{1}\left(\bar{R} \times\left[0, t_{0}\right]\right) \text {, } \\
& 0 \mathrm{R}
\end{aligned}
$$

which in turn necessitates ${ }^{2}$ that $\underset{\sim}{\mathrm{v}}$ and $\underset{\sim}{\mathrm{v}^{\prime}}$ coincide almost everywhere on $\bar{R} \times\left[0, t_{0}\right]$. Similarly, the generalized gradient of $p$ is unique in the foregoing sense. Moreover, if $\underset{\sim}{q} \in \mathbb{C}^{1}\left(\bar{R} \times\left[0, t_{0}\right]\right)$ is a vector field, it is

${ }^{1}$ Following conventional usage, we call the closure of the set of all points at which a function does not vanish the support of the function.

2 This is the vectorial version of a well-known theorem in the theory of Lebesgue integration (cf., Theorem 5 in Section 71 of Smirnov [16]). 
easily seen through an integration by parts and an appeal to the divergence theorem that the time-derivative $\underset{\sim}{\dot{q}}$ and the gradient $\nabla \underset{\sim}{q}$ are also the generalized time-derivative and the generalized gradient of q. In the event that a vector field $\underset{\sim}{\mathrm{p}}$ possesses a generalized timederivative $\underset{\sim}{v}$ and a generalized gradient $\underset{\sim}{\mathrm{w}}$, we adopt the notation $\underset{\sim}{\dot{p}}$ for $\underset{\sim}{\mathrm{v}} \partial \underset{\sim}{\mathrm{p}}$ for $\underset{\sim}{\mathrm{w}}$ and write $\dot{\partial} \mathrm{p}_{i}, \partial_{j} \mathrm{p}_{i}$ for $\mathrm{v}_{i}, w_{i j}$, respectively.

If $R$ again denotes a bounded domain in $E$, we write $\omega_{2}^{1}\left(\bar{R} \times\left[0, t_{0}\right]\right)$ for the assembly of all vector-valued functions in $\mathcal{L}_{2}\left(\bar{R} \times\left[0, t_{0}\right]\right)$ that have generalized time-derivatives and generalized gradients belonging ${ }^{1}$ to $\mathcal{L}_{2}\left(\overline{\mathrm{R}} \times\left[0, \mathrm{t}_{0}\right]\right)$. The function space $w_{2}^{1}\left(\overline{\mathrm{R}} \times\left[0, \mathrm{t}_{0}\right]\right)$ is a normed linear space with the norm

$$
\|\underline{R}\|_{R \times\left(0, t_{0}\right)}=\left\{\int_{0}^{t} \int_{R}\left(|\underset{\sim}{p}|^{2}+|\dot{\sim} \underset{\sim}{p}|^{2}+|\partial \underset{\sim}{p}|^{2}\right) d V d t\right\}^{1 / 2} .
$$

To motivate the definition of a weak elastodynamic state about to be introduced, we show first that if $\mathrm{R}$ is a regular region and

$$
\mathrm{S}=[\underset{\sim}{\mathrm{u}}, \underset{\sim}{\sigma}] \in \mathcal{E}\left(\underset{\sim}{\mathrm{f}}, \rho, \underset{\sim}{\mathrm{c}} ; \overline{\mathrm{R}} \times\left[0, \mathrm{t}_{\mathrm{o}}\right]\right),
$$

then, for every $\underset{\sim}{\varphi} \in \mathrm{C}^{1}\left(\overline{\mathrm{R}} \times\left[0, \mathrm{t}_{\mathrm{o}}\right]\right)$,

$$
\begin{aligned}
& \int_{0 R}^{t} \int_{R}\left(o \dot{\varphi}_{i} \dot{u}_{i}-\varphi_{i, j} \sigma_{i j}+\varphi_{i} f_{i}\right) d V d t+\int_{0}^{o} \int_{\partial R} \varphi_{i} s_{i} d A d t \\
& +\int_{R} \rho(\underset{\sim}{x})\left(\varphi_{i}(\underset{\sim}{x}, 0) \dot{u}_{i}(\underset{\sim}{x}, 0)-\varphi_{i}\left(\underset{\sim}{x}, t_{0}\right) \dot{u}_{i}\left(\underset{\sim}{x}, t_{0}\right)\right) d V=0 .
\end{aligned}
$$

$1_{\text {Thus, }} w_{2}^{1}\left(\bar{R} \times\left[0, t_{0}\right]\right)$ is a "Sobolev space" of vector fields (see [4 7). An exposition of the theory of generalized derivatives and Sobolev spaces is also given, for example, in Smirnov [16]. 
To prove this identity, let $\underset{\sim}{\varphi} \mathrm{C}^{1}\left(\overline{\mathrm{R}} \times\left[0, \mathrm{t}_{0}\right]\right),\left[\mathrm{t}_{1}, \mathrm{t}_{2}\right] \subset\left(0, \mathrm{t}_{0}\right)$ and define a vector field $\underset{\sim}{p}$ on $\bar{R}$ through

$$
\left.\left.\left.\mathrm{p}_{\mathrm{j}} \underset{\sim}{\mathrm{x}}\right)=-\int_{\mathrm{t}_{1}}^{\mathrm{t}_{2}} \varphi_{\mathrm{i}} \underset{\sim}{\mathrm{x}}, \mathrm{t}\right) \sigma_{\mathrm{ij}} \underset{\sim}{(\mathrm{x}}, \mathrm{t}\right) \mathrm{dt} \text { for all } \underset{\sim}{\mathrm{x}} \in \overline{\mathrm{R}} .
$$

Next, use the stress equations of motion (1.7) to see that

$$
\begin{aligned}
& p_{j, j}(\underset{\sim}{x})=\int_{t}^{t_{2}}\left(\rho(\underset{\sim}{x}) \dot{\varphi}_{i}(\underset{\sim}{x}, t) \dot{u}_{i}(\underset{\sim}{x}, t)-\varphi_{i, j}(\underset{\sim}{x}, t) \sigma_{i j}(\underset{\sim}{x}, t)+\varphi_{i}(\underset{\sim}{x}, t) f_{i}(\underset{\sim}{x}, t)\right) d t \\
& +\varphi_{i}\left(\underset{\sim}{x}, t_{1}\right) \dot{u}_{i}\left(\underset{\sim}{x}, t_{1}\right)-\varphi_{i}\left(\underset{\sim}{x}, t_{2}\right) \dot{u}_{i}\left(\underset{\sim}{x}, t_{2}\right) \text { for every } \underset{\sim}{x \in R} .
\end{aligned}
$$

Thus,

$$
p \in C^{1}(R) \cap C(\bar{R}) \quad, \quad \nabla \cdot \underset{\sim}{p} \in C(\bar{R}) .
$$

Applying the divergence theorem to $\underset{\sim}{p}$ on $\bar{R}$ and then letting $t_{1}, t_{2}$ tend to $0, t_{0}$ respectively, we obtain (3.4).

Definition 3. 1. (Weak elastodynamic state). Let $R,\left(0, t_{0}\right), \rho \underline{\text { and }} \underset{\sim}{\mathrm{c}}$ be as in Definition 1.1 and let $\underset{\sim}{f}$ be a vector field, measurable on $\bar{R} \times\left[0, t_{0}\right]$. Then, $S=[\underset{\sim}{\sim} \underset{\sim}{\sigma}]$ is said to be a weak elastodynamic state on $\overline{\mathrm{R}} \times\left[0, \mathrm{t}_{\mathrm{O}}\right]$ corresponding to the body-force field $\underset{\sim}{f}$, the mass-density field

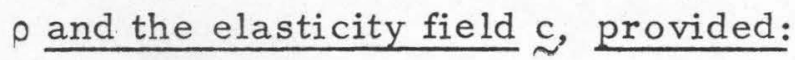

$$
\begin{aligned}
& \text { (i) } \underset{\sim}{f} \in \mathcal{L}_{2}\left(\overline{\mathrm{R}} \times\left[0, \mathrm{t}_{\mathrm{o}}\right]\right), \underset{\sim}{u} \in w_{2}^{1}\left(\overline{\mathrm{R}} \times\left[0, \mathrm{t}_{0}\right]\right) . \\
& \text { (ii) } \sigma_{i j}=c_{i j k \ell} \partial_{\ell} \mathrm{u}_{\mathrm{k}} \text { on } \overline{\mathrm{R}} \times\left[0, \mathrm{t}_{\mathrm{o}}\right],
\end{aligned}
$$

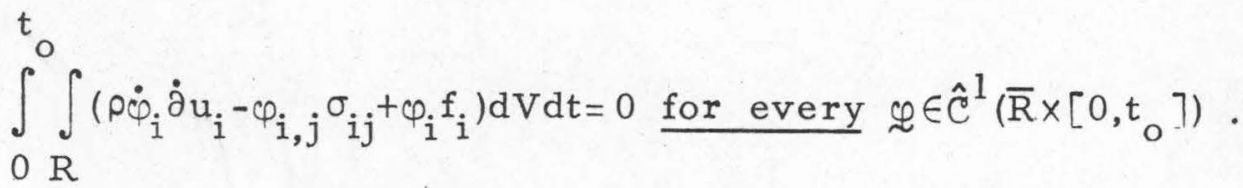


For the collection of all such states, we write

$$
\mathcal{E}_{\mathrm{w}}\left(\underset{\sim}{\mathrm{f}}, \rho, \mathrm{c} ; \overline{\mathrm{R}} \times\left[0, \mathrm{t}_{\mathrm{O}}\right]\right) \text {. }
$$

Observe that if

$$
\left.S=[\underset{\sim}{\mathrm{u}} \underset{\sim}{\sigma}] \in \varepsilon_{\mathrm{w}} \underset{\sim}{f}, \rho, \underset{\sim}{\mathrm{c}} ; \overline{\mathrm{R}} \times\left[0, \mathrm{t}_{\mathrm{o}}\right]\right),
$$

$\underset{\sim}{\sigma} \in \mathcal{L}_{2}\left(\overline{\mathrm{R}} \times\left[0, \mathrm{t}_{\mathrm{o}}\right]\right)$ because of $(3.5)$ and the required regularity of $\underset{\sim}{\mathrm{u}}$ and $\underset{\sim}{\mathrm{c}}$. Further, the concept of a weak elastodynamic state is an extension of the notion of a regular elastodynamic state. Indeed, if

$$
S=[\underset{\sim}{\mathrm{u}} \underset{\sim}{\sigma}] \in \mathcal{\varepsilon}\left(\underset{\sim}{\mathrm{f}}, \rho, \mathrm{c} ; \overline{\mathrm{R}} \times\left[0, \mathrm{t}_{\mathrm{o}}\right]\right),
$$

then Definition 1.1 and the identity ${ }^{l}$ (3.4) with $\underset{\sim}{\varphi} \hat{\mathrm{C}}^{1}\left(\overline{\mathrm{R}} \times\left[0, \mathrm{t}_{0}\right]\right)$ imply that

$$
s \in \varepsilon_{\mathrm{w}}\left(\underset{\sim}{f}, \rho, \mathrm{c} ; \overline{\mathrm{R}} \times\left[0, \mathrm{t}_{\mathrm{o}}\right]\right) .
$$

Conversely, suppose

$$
S=[\underset{\sim}{\mathrm{u}}, \underset{\sim}{\sigma}] \in \varepsilon_{\mathrm{w}}\left(\underset{\sim}{\mathrm{f}}, \rho, \underset{\sim}{\mathrm{c}} ; \overline{\mathrm{R}} \times\left[0, \mathrm{t}_{\mathrm{o}}\right]\right),
$$

such that

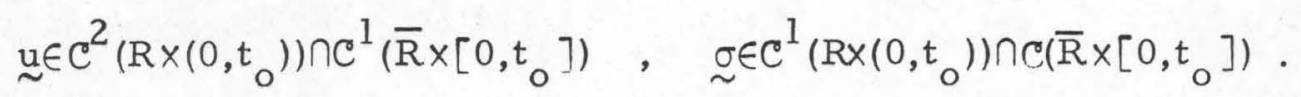

Moreover, assume that the three functions $\sigma_{i j, j}-p \ddot{q}_{i}$ are continuous on $\overline{\mathrm{R}} \times\left[0, \mathrm{t}_{0}\right]$. Then

$$
\mathrm{s} \in \mathcal{E}\left(\underset{\sim}{f^{\prime}}, \rho, \underset{\sim}{c} ; \overline{\mathrm{R}} \times\left[0, \mathrm{t}_{\mathrm{o}}\right]\right),
$$

where

${ }^{1}$ When $\mathscr{Q} \in \hat{C}^{1}\left(\bar{R} \times\left[0, t_{0}\right]\right)$, Equation (3.4) is valid for any domain $R$ in $E$. To see this, enclose $\bar{R}$ within a regular region $R$, extend $\varphi$ and $S$ to $\bar{R}$ by letting them vanish on $\bar{R}-\bar{R}$, and apply the argument used earlier in connection with the proof of (3.4) to the extended vector field $\mathrm{Q}$ on $\bar{R}$ (see the discussion following (3.4)). 


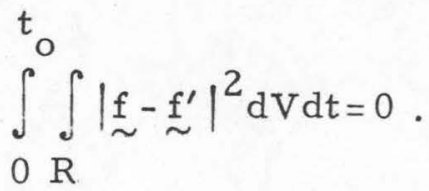

To verify this claim, define $\underset{\sim}{f^{\prime}}$ on $\bar{R} \times\left[0, t_{0}\right]$ through

$$
f_{i}^{\prime}=\rho \ddot{u}_{i}-\sigma_{i j, j} \text { on } \bar{R} \times\left[0, t_{0}\right] \text {, }
$$

so that (*) follows at once from Definition 3.1 and the assumed regularity of $\underset{\sim}{u}$ and $\underset{\sim}{\sigma}$. Thus,

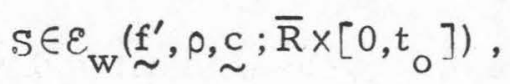

whence (3.5) also holds with $\underset{\sim}{f}$ replaced by $\underset{\sim}{f} . \quad$ Therefore,

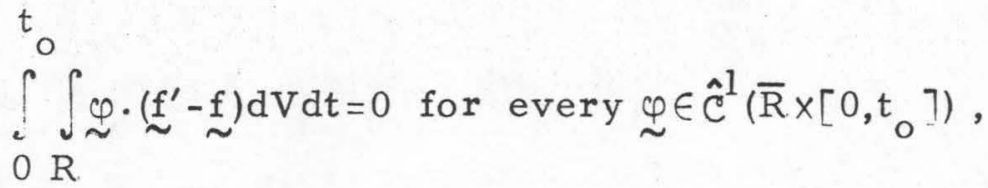

from which (**) follows.

In preparation for a suitable generalization of the initial and boundary conditions (1.9), (1.10), we introduce three subclasses of $w_{2}^{1}\left(\bar{R} \times\left[0, t_{0}\right]\right)$ through

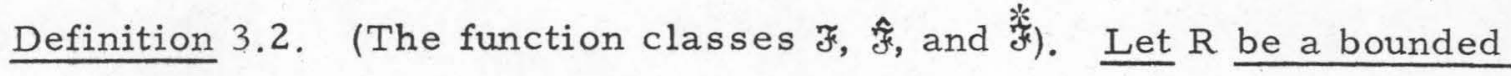
domain in $\mathrm{E}$ and $\left(0, \mathrm{t}_{\mathrm{o}}\right)$ a time-interval. Then, we write:

(i) $\mathrm{p} \in \mathfrak{G}\left(\overline{\mathrm{R}} \times\left[0, \mathrm{t}_{0}\right]\right)$, provided $\underset{\sim}{\mathrm{p}} \in \omega_{2}^{1}\left(\overline{\mathrm{R}} \times\left[0, \mathrm{t}_{0}\right]\right)$ and there exists a sequence $\left\{\mathrm{p}^{\mathrm{n}}\right\}$ of vector fields such that

$$
\begin{aligned}
& \underset{\sim}{\mathrm{p}} \in \mathrm{C}^{1}\left(\overline{\mathrm{R}} \times\left[0, \mathrm{t}_{0}\right]\right) \quad(\mathrm{n}=1,2, \cdots), \\
& \lim _{n \rightarrow \infty}\left\|\underline{\sim}-\sim_{\sim}^{n}\right\|_{R \times\left(0, t_{0}\right)}= \\
& \lim _{n \rightarrow \infty}\left\{\int_{0}^{t} \int_{R}\left(\left|\underset{\sim}{\mid p-p^{n}}\right|^{2}+\left|\dot{\partial} \underset{\sim}{p}-\dot{\sim}^{n}\right|^{2}+|\partial \underset{\sim}{p}-\nabla \underset{\sim}{n}|^{2}\right) d v d t\right\}^{1 / 2}=0 .
\end{aligned}
$$


(ii) $\mathrm{p} \in \hat{\mathfrak{G}}\left(\overline{\mathrm{R}} \times\left[0, \mathrm{t}_{0}\right]\right)$, if $\underset{\sim}{\mathrm{p}} \in \mathrm{C}^{1}\left(\overline{\mathrm{R}} \times\left[0, \mathrm{t}_{0}\right]\right)$ is a vector field and there exists $8>0$, possibly depending on $p$, such that

$$
\mathrm{p}=\underset{\sim}{0} \text { on } \overline{\mathrm{R}} \times\left(\mathrm{t}_{\mathrm{o}}-\delta, \mathrm{t}_{\mathrm{o}}\right] \text {. }
$$

(iii) $\underset{\sim}{\mathrm{p}} \in \underset{*}{*}\left(\overline{\mathrm{R}} \times\left[0, \mathrm{t}_{0}\right]\right)$, if $\underset{\sim}{\mathrm{p}} \in \mathrm{u}_{2}^{1}\left(\overline{\mathrm{R}} \times\left[0, \mathrm{t}_{\mathrm{o}}\right]\right)$ and there exists a sequence $\left\{\underline{\mathrm{p}}^{\mathrm{n}}\right\}$ of vector fields such that

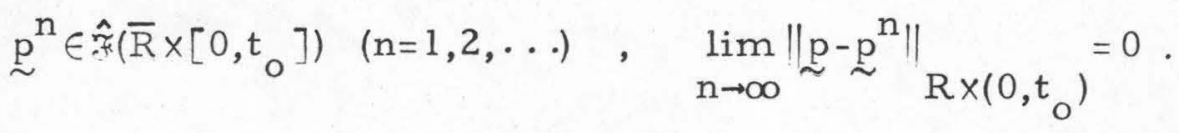

Thus, $F\left(\bar{R} \times\left[0, t_{0} 7\right)\right.$ is the closure in the $w_{2}^{l}$-norm of the set of all vector fields belonging to $\mathrm{C}^{1}\left(\overline{\mathrm{R}} \times\left[0, \mathrm{t}_{0}\right]\right)$. On the other hand, 霄 $\left(\overline{\mathrm{R}} \times\left[0, \mathrm{t}_{0}\right]\right)$ is the closure in the $w_{2}^{1}$-norm of the set $\hat{\mathfrak{F}}\left(\overline{\mathrm{R}} \times\left[0, \mathrm{t}_{0}\right]\right)$ of all vector fields in $C^{1}\left(\bar{R} \times\left[0, t_{0}\right]\right)$ that vanish identically on $\bar{R}$ near the time $t_{0}$. Since $w_{2}^{1}$ is complete (cf., Smirnov [16], Section 112), 类 can be viewed alternatively as the completion in the $w_{2}^{1}$-norm of the class $\hat{G}$ (see Smirnov [16], Section 85, for example), an analogous remark being in order for 3 . Notice also that

$$
\hat{\mathfrak{F}}\left(\overline{\mathrm{R}} \times\left[0, \mathrm{t}_{0}\right]\right) \subset \underset{\mathfrak{*}}{*}\left(\overline{\mathrm{R}} \times\left[0, \mathrm{t}_{0}\right]\right) \subset \mathfrak{\xi}\left(\overline{\mathrm{R}} \times\left[0, \mathrm{t}_{0}\right]\right) \subset w_{2}^{1}\left(\overline{\mathrm{R}} \times\left[0, \mathrm{t}_{0}\right]\right) .
$$

For a wide class of bounded domains in $E, F\left(\bar{R} \times\left[0, t_{0}\right]\right)$ is in fact all of $w_{2}^{1}\left(\bar{R} \times\left[0, t_{0}\right]\right)$. The counterpart of this result for bounded domains in $E_{n}$ was apparently first established by Friedrichs [17] under certain restrictions as to the nature of the domain. Moreover, the foregoing result is known to hold for bounded, star-shaped domains ${ }^{1}$ in $E_{n}$. In what follows we prove the equivalence of $\bar{F}\left(\bar{R} \times\left[0, t_{0}\right]\right)$ and $w_{2}^{1}\left(\bar{R} \times\left[0, t_{0}\right]\right)$ under the weaker assumption that $R$ is merely locally 
star-shaped $^{1}$; i. e., we suppose that for every $\underset{\sim}{x} \in \overline{\mathrm{R}}$ there exists a neighborhood $\mathrm{N}(\underset{\sim}{\mathrm{x}})$ of $\underset{\sim}{\mathrm{x}}$ such that $\mathrm{N}(\underset{\sim}{\mathrm{x}}) \cap \mathrm{R}$ is star-shaped. Observe that a bounded, locally star-shaped domain is necessarily the union of a finite collection of star-shaped domains.

Theorem 3.1. Let $\mathrm{R}$ be a bounded, locally star-shaped domain in $\mathrm{E}$. Then,

$$
\xi\left(\bar{R} \times\left[0, t_{0}\right]\right)=w_{2}^{1}\left(\bar{R} \times\left[0, t_{0}\right]\right) .
$$

Proof: It suffices to show that

$$
\omega_{2}^{1}\left(\bar{R} \times\left[0, t_{0}\right]\right) \subset \mathfrak{z}\left(\bar{R} \times\left[0, t_{0}\right]\right) .
$$

For every $\underset{\sim}{x} \in \bar{R}$, choose a neighborhood $N(\underset{\sim}{x})$ of $\underset{\sim}{x}$ such that $N(\underset{\sim}{x}) \cap R$ is star-shaped. Since $\overline{\mathrm{R}}$ is compact, there exists a finite point set $\left\{\underset{\sim}{x^{1}}, \underset{\sim}{2}, \cdots, \underset{\sim}{x^{m}}\right\}$ in $\bar{R}$ such that

$$
\bigcup_{k=1}^{m} N\left(x^{k}\right) \supset \bar{R}
$$

Next, let

$$
\mathrm{N}_{\mathrm{k}}=\mathrm{N}(\underset{\sim}{\mathrm{x}}) \quad(\mathrm{k}=1, \cdots, \mathrm{m}),
$$

let $\mathrm{T}$ be an open interval containing $\left[0, \mathrm{t}_{0}\right]$, and set

$$
\begin{array}{cc}
\mathrm{D}=\mathrm{R} \times\left(0, \mathrm{t}_{\mathrm{o}}\right), \quad \mathrm{Q}_{\mathrm{k}}=\mathrm{N}_{\mathrm{k}} \times \mathrm{T} \quad(\mathrm{k}=1, \cdots, \mathrm{m}), \\
\mathrm{D}_{\mathrm{k}}=\mathrm{Q}_{\mathrm{k}} \cap \mathrm{D}=\left(\mathrm{N}_{\mathrm{k}} \cap \mathrm{R}\right) \times\left(0, \mathrm{t}_{\mathrm{o}}\right) \quad(\mathrm{k}=1, \cdots, \mathrm{m}),
\end{array}
$$

$\overline{1}$ This equivalence is established by Agmon [18] (p. 11) for (not necessarily bounded) domains that have the "s egment property". 
whence each $D_{k}$ is star-shaped and

$$
\bigcup_{\mathrm{k}=1}^{\mathrm{m}} \mathrm{Q}_{\mathrm{k}} \supset \overline{\mathrm{R}} \times\left[0, \mathrm{t}_{\mathrm{o}}\right] \text {. }
$$

Suppose now that $\underset{\sim}{\mathrm{p}} \in w_{2}^{1}(\overline{\mathrm{D}})$. Then $\underset{\sim}{\mathrm{p}} \in w_{2}^{1}\left(\overline{\mathrm{D}}_{\mathrm{k}}\right)(\mathrm{k}=1, \ldots, \mathrm{m})$, and one has from the well-known result cited earlier (cf., Smirnov [16], Section 111$)$ that $\underset{\sim}{\mathrm{p}} \in \mathfrak{F}\left(\overline{\mathrm{D}}_{\mathrm{k}}\right)(\mathrm{k}=1, \ldots, \mathrm{m})$. Thus, for each $\mathrm{k}$, there exists a sequence $\left\{\mathrm{p}_{\mathrm{k}}^{\mathrm{n}}\right\}$ of vector-valued functions such that

$$
\underset{\sim}{p_{k}} \in C^{1}\left(\bar{D}_{k}\right) \quad(n=1,2, \cdots) \quad, \quad \lim _{n \rightarrow \infty}\left\|\underset{\sim}{p}-\underset{\sim}{p_{k}^{n}}\right\|_{D_{k}}=0
$$

Introduce a partition of unity $\left\{\psi_{1}, \psi_{2}, \ldots, \psi_{\mathrm{m}}\right\}$ subordinate to $\left\{Q_{1}, Q_{2}, \cdots, Q_{m}\right\}$ by requiring that

$$
\begin{aligned}
& \psi_{k} \in C^{\infty}\left(E_{4}\right) \quad(k=1, \cdots, m), \\
& \sum_{k=1}^{m} \psi_{k} \underset{\sim}{(x, t)}=1 \text { for every } \underset{\sim}{(x, t) \in D,} \quad \Omega_{k} \subset Q_{k}(k=1, \ldots, m),
\end{aligned}
$$

where $\Omega_{\mathrm{k}}$ is the support of $\psi_{\mathrm{k}}$. Further, let

$$
\underset{\sim}{\mathrm{q}_{\mathrm{k}}^{\mathrm{n}}}=\left\{\begin{array}{l}
\psi_{\mathrm{k}} \mathrm{p}_{\mathrm{k}}^{\mathrm{n}} \text { on } \mathrm{D}_{\mathrm{k}} \\
\underset{\sim}{0} \text { on } \overline{\mathrm{D}}-\mathrm{D}_{\mathrm{k}}
\end{array} \quad(\mathrm{k}=1, \ldots, \mathrm{m} ; \mathrm{n}=1,2, \ldots), \quad \text { (no sum on } \mathrm{k}\right. \text { ). }
$$

From (C), (D) and (E) follows

$$
\mathrm{q}_{\mathrm{k}}^{\mathrm{n}} \in \mathrm{C}^{1}(\overline{\mathrm{D}}) \quad(\mathrm{k}=1, \cdots, \mathrm{m} ; \mathrm{n}=1,2, \cdots) .
$$

\footnotetext{
${ }^{1}$ For a proof of the existence of a partition of unity subordinate to a given covering, refer, for instance, to Bremermann [19] (Section 3.6). Partitions of unity have frequently been used in similar contexts (e.g., see Friedman [20], Chapter 10).
} 
Thus, if

$$
\underset{\sim}{\mathrm{p}}=\sum_{\mathrm{k}=1}^{\mathrm{m}} \underset{\mathrm{q}}{\mathrm{q}} \text { on } \overline{\mathrm{D}} \quad(\mathrm{n}=1,2, \cdots)
$$

(F) reveals that

$$
\mathrm{e}^{\mathrm{n}} \in \mathrm{C}^{1}(\overline{\mathrm{D}}) \quad(\mathrm{n}=1,2, \cdots) .
$$

Moreover, using (D), (G) and the triangle inequality, one has

$$
\left\|\underset{\sim}{\mathrm{p}}-\mathrm{p}_{\sim}^{\mathrm{n}}\right\|_{\mathrm{D}} \leq \sum_{\mathrm{k}=1}^{\mathrm{m}}\left\|\psi_{\mathrm{k} \sim} \mathrm{p}-\mathrm{q}_{\sim}^{\mathrm{n}}\right\|_{D}(\mathrm{n}=1,2, \cdots)
$$

But, from (D) and (E),

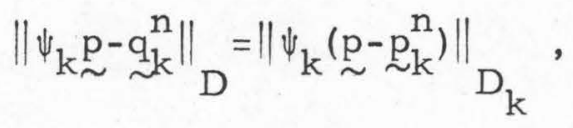

and since for any pair of tensors $\underset{\sim}{a}$ and $\underset{\sim}{b}$ of the same order,

$$
|\underset{\sim}{a}+\underset{\sim}{b}|^{2} \leq 2\left(|\underset{\sim}{a}|^{2}+|\underset{\sim}{b}|^{2}\right)
$$

one infers from $(\mathrm{J})$ that ${ }^{1}$

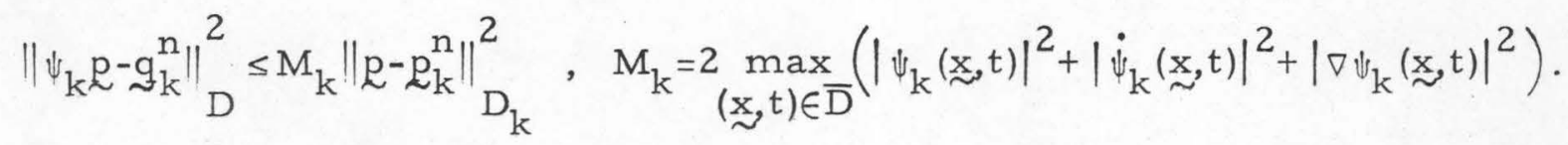

Thus, because of (I),

$$
\|\underset{\sim}{\mathrm{p}}-\underset{\mathrm{p}}{\mathrm{n}}\|_{\mathrm{D}} \leq \mathrm{M} \sum_{\mathrm{k}=1}^{\mathrm{m}} \| \underset{\sim}{\mathrm{p}}-\underset{\mathrm{p}_{\mathrm{k}}^{\mathrm{n}}}{\mathrm{n}_{\mathrm{D}_{\mathrm{k}}}}(\mathrm{n}=1,2, \cdots) \quad, \quad \mathrm{M}=\max _{1 \leq \mathrm{k} \leq \mathrm{m}} \sqrt{\mathrm{M}_{\mathrm{k}}} .
$$

Equations (C) and (K), together with (H), imply ${ }_{\text {Recall that the product rule applies to generalized differentiation of }}$
functions in $10_{2}^{1}$ (cf., Smirnov [16], Section 109). 


$$
\underset{\sim}{p} \in \mathfrak{F}(\bar{D}),
$$

whence (A) holds, and the proof is complete.

It can be shown (see, for instance, Smirnov [16], Sections 113 and 110$)$ that if $\underset{\sim}{p} \in \mathcal{F}\left(\bar{R} \times\left[0, t_{0}\right]\right)$, there exists $\underset{\sim}{q} \in \mathcal{J}\left(\bar{R} \times\left[0, t_{0}\right]\right)$, coincident with $\underset{\sim}{p}$ almost everywher $e^{1}$, such that for all $t \in\left[0, t_{0}\right]$ :

$$
\begin{aligned}
& \underset{\sim}{q}(\cdot, t) \in \delta_{2}(\bar{R}), \quad \lim _{\delta \rightarrow 0} \int_{R}|\underset{\sim}{q}(\underset{\sim}{x}, t+\delta)-\underset{\sim}{q}(x, t)|^{2} d V=0, \\
& \underset{\sim}{q}(\underset{\sim}{x}, t)=\underset{\sim}{q}(\underset{\sim}{x}, 0)+\int_{0}^{t} \partial \underset{\sim}{q}(\underset{\sim}{x}, \tau) d \tau \text { for almost all } \underset{\sim}{x \in R},
\end{aligned}
$$

We therefore ascribe these properties to every element of 3 and, through Theorem 3.1 , to every member of $w_{2}^{1}$, provided $R$ is locally star-shaped.

Motivated by the foregoing remark and by the identity (3.4), we formulate the traction problem for weak elastodynamic states as follows. Given a regular, locally star-shaped region $\mathrm{R}$, and a timeinterval $\left(0, t_{0}\right)$, find

$$
\mathrm{S}=[\underset{\sim}{\mathrm{u}}, \underset{\sim}{\sigma}] \in \varepsilon_{\mathrm{w}}(\underset{\sim}{f}, \rho, \mathrm{c} ; \overline{\mathrm{R}} \times[0, \mathrm{t},])
$$

corresponding to the data $\underset{\sim}{f}, 0, \underset{\sim}{c}$ and subject to the generalized boundary and initial conditions

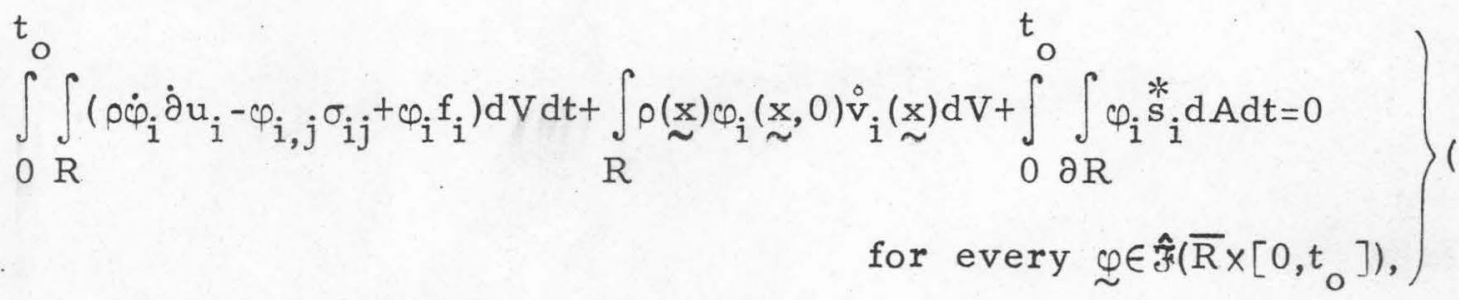

I Observe that if two functions belonging to $w_{2}^{1}$ coincide almost every where, then (3.1) and (3.2) assure that they have the same generalized time-derivative and the same generalized gradient. 


$$
\left.\lim _{t \rightarrow 0} \int_{R} \mid \underset{\sim}{u} \underset{\sim}{x}, t\right)-\left.\underset{\sim}{\sim}(\underset{\sim}{x})\right|^{2} d V=0,
$$

where $\stackrel{\circ}{\sim}, \stackrel{\circ}{\sim}$ and $\underset{\sim}{\stackrel{*}{\sim}}$ are prescribed vector-valued functions, squaresummable on $\bar{R}, \bar{R}$, and $\partial \mathrm{R} \times\left[0, t_{0}\right]$, respectively.

Equations (3.8), (3.9) constitute a generalization of (1.9), (1.10), the second of (1.9) being incorporated with (1.10) in (3.8). Indeed, if $\mathrm{R}$ is a regular region and $\stackrel{\stackrel{\sim}{\sim}}{\stackrel{\sim}{\sim}}, \underset{\sim}{\stackrel{*}{\sim}}$ are vector fields obeying

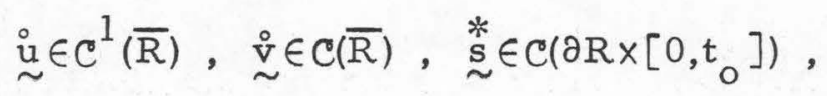

while

$$
\mathrm{S}=[\underset{\sim}{\mathrm{u}}, \underset{\sim}{\sigma}] \in \mathcal{E}\left(\underset{\sim}{\mathrm{f}}, \rho, \mathrm{c} ; \overline{\mathrm{R}} \times\left[0, \mathrm{t}_{\mathrm{o}}\right]\right) \text {, }
$$

then (3.8) and (3.9) are equivalent to (1.9) and (1.10). We show only that (3.8), (3.9) imply (1.9), (1.10), since the converse is clear from (*), $(* *)$, and (3.4). As a consequence of (*), (**), (3.4), (3.8), and (3.9),

$$
\underset{\sim}{\mathrm{u}}(\cdot, 0)=\stackrel{\circ}{\sim} \text { on } \overline{\mathrm{R}} \text {, }
$$

whereas for every $\underset{\sim}{\varphi} \in \hat{\mathfrak{k}}\left(\overline{\mathrm{R}} \times\left[0, \mathrm{t}_{\mathrm{O}}\right]\right)$,

$$
\int_{R} \rho(\underset{\sim}{x}) \varphi_{i}(\underset{\sim}{x}, 0)\left(\dot{u}_{i}(\underset{\sim}{x}, 0)-\dot{v}_{i}(\underset{\sim}{x}, 0)\right) d V+\int_{0}^{t_{\partial R}} \int_{\partial R} \varphi_{i}\left(s_{i}-\stackrel{*}{s}_{i}\right) d A d t=0 .
$$

Thus, appealing to the appropriate lemmas from the calculus of variations, one concludes from $(\neq)$ that

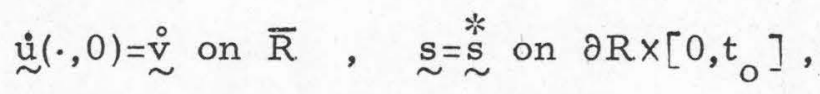

which, together with $(\uparrow)$, yield (1.9), (1.10).

We turn now to a lemma which will be used in the proof of a subsequent uniqueness theorem associated with the traction problem for weak elastodynamic states. 
Lemma 3.1. Let $\mathrm{R}$ be a bounded, locally star-shaped domain in $\mathrm{E}$. Suppose that $\underset{\sim}{\mathrm{p}} \in w_{2}^{1}\left(\overline{\mathrm{R}} \times\left[0, \mathrm{t}_{0}\right]\right), \mathrm{t}_{1} \in\left[0, \mathrm{t}_{0}\right)$ and let

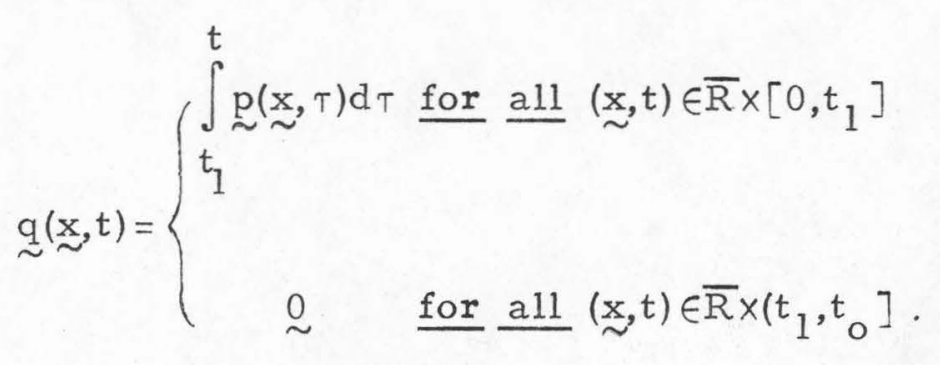

Then, $\underset{\sim}{q} \in \underset{\vec{*}}{*}\left(\overline{\mathrm{R}} \times\left[0, t_{0}\right]\right)$ and its generalized time-derivative and generalized gradient are given by

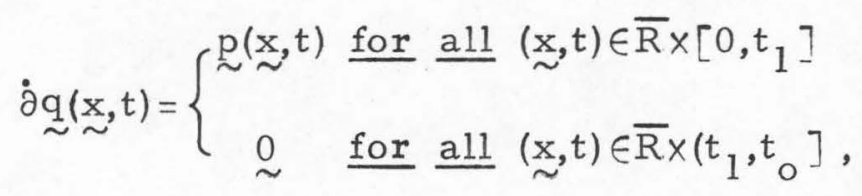

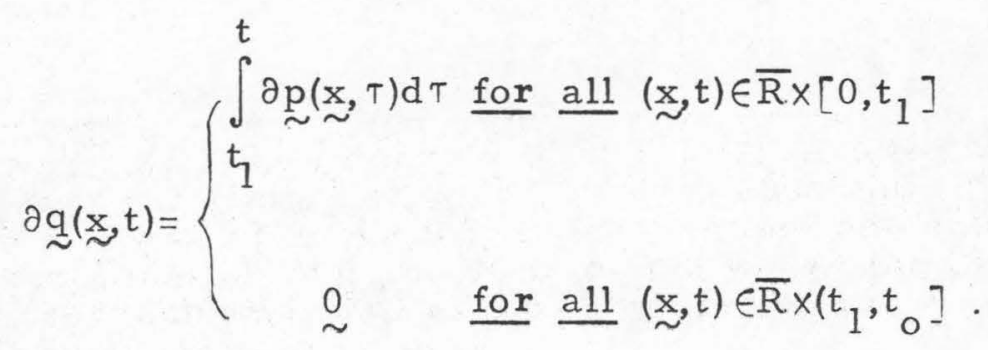

Proof: Observe from (3.10) that there exists $\delta>0$ such that

$$
\underset{\sim}{\mathrm{q}}=\underset{\sim}{0} \text { on } \overline{\mathrm{R}} \times\left(\mathrm{t}_{\mathrm{o}}-\delta, \mathrm{t}_{\mathrm{o}}\right] \text {. }
$$

Suppose first, as will subsequently be confirmed, that

$$
\underset{\sim}{q} \in w_{2}^{1}\left(\bar{R} \times\left[0, t_{0}\right]\right) \text {. }
$$

We claim that (A), (B) and the hypotheses on $R$ imply

${ }_{\text {For fixed }} t \in\left[0, t_{1}\right)$, the integrals in (3.10), (3.12) need not exist for all $x \in \bar{R}$, but only for almost all $x \in \bar{R}$. Strictly speaking, a complete definition of $q$ and $\partial \mathrm{q}$ on $\overline{\mathrm{R}} \times[\tilde{0, t}]$ requires that one adjoin to $(3.10)$, (3.12) a specification of $\mathrm{q}$ and $\partial \mathrm{q}$ at points where the time-integrals of $\mathrm{p}$ and $\partial \mathrm{p}$, respectively, fail to exist; in particular, $\mathrm{q}$ and $\underset{\sim}{\mathrm{q}}$ could be taken tõ be zero at these points. 


$$
\underset{\sim}{q} \in \underset{3}{*}\left(\bar{R} \times\left[0, t_{0}\right]\right)
$$

To see this, note that if $R$ were star-shaped (rather than locally starshaped), (A) and (B) would be sufficient for (C), since the requisite sequence $^{l}$ of vector fields in $\hat{\mathfrak{z}}\left(\overline{\mathrm{R}} \times\left[0, \mathrm{t}_{0}\right]\right)$ tending to $\underset{\sim}{\mathrm{q}}$ in the norm of $w_{2}^{1}$ could be constructed with the aid of the scheme employed by Smirnov [16] (Section 111) in a similar context. The conclusion (C) for a merely locally star-shaped $R$ may now be verified through an argument analogous to the one used in the proof of Theorem 3.1.

It remains to be shown that (B), (3.11), and (3.12) hold true. With this objective in mind, suppose $\underset{\sim}{\varphi} \in \hat{C}^{1}\left(\overline{\mathrm{R}} \times\left[0, \mathrm{t}_{\mathrm{O}}\right]\right)$. Then, (3.10) yields

$$
\begin{aligned}
& \int_{0}^{t_{0}} \int_{R} \dot{\varphi}_{i} q_{i} d V d t=\int_{0}^{t_{R}} \int_{R}\left(\dot{\varphi}_{i} \underset{\sim}{(x, t)} \int_{t_{1}}^{t} p_{i}(\underset{\sim}{x}, \tau) d \tau\right) d V d t, \\
& \int_{0}^{t_{0}} \int_{R}^{\varphi_{i}} \varphi_{i, j} q_{i} d V d t=\int_{0}^{t_{1}} \int_{R}\left(\varphi_{i, j} \underset{\sim}{(x, t)} \int_{t_{1}}^{t} p_{i} \underset{\sim}{(x, \tau) d \tau}\right) d V d t .
\end{aligned}
$$

But, according to the formula for integration by parts (cf., McShane and Botts [21], p. 191),

$$
\int_{0}^{t_{1}}\left(\dot{\varphi}_{i}(\underset{\sim}{x}, t) \int_{t_{1}}^{t} p_{i}(x, \tau) d \tau\right) d t=-\int_{0}^{t_{1}} \varphi_{i}(\underset{\sim}{x}, t) p_{i}(\underset{\sim}{x}, t) d t \text { for almost all } \underset{\sim}{x} \in \bar{R} .
$$

Integrating both members of $(F)$ over $R$, interchanging the order of integration, and using (D), one arrives at 


$$
\int_{0}^{t_{0}} \int_{R} \dot{\varphi}_{i} q_{i} d V d t=-\int_{0}^{t_{R}} \int_{R} \varphi_{i} p_{i} d V d t
$$

so that $\underset{\sim}{q}$ has a generalized time-derivative given by (3.11).

The analogous procedure applied to $(E)$ leads to

$$
\int_{0}^{t_{0}} \int_{R} \varphi_{i, j} q_{i} d V d t=-\int_{0}^{t_{1}} \int_{R}\left(p_{i} \underset{\sim}{(x, t)} \int_{0}^{t} \varphi_{i, j} \underset{\sim}{(x, \tau) d \tau) d V d t .}\right.
$$

We show next that since $\underset{\sim}{p} \in w_{2}^{1}\left(\bar{R} \times\left[0, t_{0}\right]\right)$,

$$
\int_{R}\left(p_{i}(x, t) \int_{0}^{t} \varphi_{i, j}(\underset{\sim}{x}, \tau) d \tau\right) d V=-\int_{R}\left(\partial_{j} p_{i}(x, t) \int_{0}^{t} \varphi_{i}(\underset{\sim}{\sim}, \tau) d \tau\right) d V
$$

for almost every $t \in\left[0, t_{0}\right]$. For this purpose, let the support of $g \in C^{1}\left(\left[0, t_{0}\right]\right)$ be contained in $\left(0, t_{0}\right)$. Then, the vector field $\underset{\sim}{\Psi}$ defined on $\bar{R} \times\left[0, t_{0}\right]$ by

$$
\left.\underset{\sim}{\Psi}(x, t)=g(t) \int_{0}^{t} \underset{\sim}{\mathscr{L}} \underset{\sim}{x}, \tau\right) \mathrm{d} \tau \text { for every } \underset{\sim}{(x, t) \in \bar{R}} \times\left[0, t_{0}\right]
$$

is in $\hat{C}^{1}\left(\overline{\mathrm{R}} \times\left[0, \mathrm{t}_{0}\right]\right)$. Taking $\underset{\sim}{\varphi}$ in $(3.2)$ to be $\underset{\sim}{\Psi}$, we infer

$$
\left.\int_{0}^{t_{0}}\left[g(t) \int_{R}\left(p_{i} \underset{\sim}{(x, t)} \int_{0}^{t} \varphi_{i, j} \underset{\sim}{(x, \tau) d \tau+\partial_{j}} \underset{i}{p_{i}} \underset{\sim}{(x, t)} \int_{0}^{t} \varphi_{i} \underset{\sim}{\underset{\sim}{x}}, \tau\right) d \tau\right) d V\right] d t=0,
$$

whence, g having been chosen arbitrarily, (H) follows. Now, integrate $(H)$ over $\left[0, t_{1}\right]$ and use the resulting identity in $(G)$ to obtain

$$
\int_{0}^{t_{0}} \int_{R} \varphi_{i, j} q_{i} d V d t=\int_{0}^{t_{1}} \int_{R}\left(\partial_{j} p_{i}(\underset{\sim}{x}, t) \int_{0}^{t} \varphi_{i}(\underset{\sim}{\sim}, \tau) d \tau\right) d V d t .
$$


Interchanging the order of integration in the right-hand member of (I), carrying out the time-integration by parts, and then restoring the former order of integration results in

$$
\left.\int_{0}^{t_{0}} \int_{R} \varphi_{i, j} q_{i} d V d t=-\int_{0}^{t_{1}} \int_{R}\left(\varphi_{i} \underset{\sim}{(x, t)} \int_{t_{1}}^{t} \partial p_{i} \underset{\sim}{(x}, \tau\right) d \tau\right) d V d t .
$$

Thus, $\underset{\sim}{q}$ has a generalized gradient given by (3.12). Finally, since $\underset{\sim}{\mathrm{p}}$ and $\partial \mathrm{p}$ belong to $\Sigma_{2}$, so do $\underset{\sim}{\mathrm{q}}, \underset{\sim}{\dot{q}}$ and $\underset{\sim}{\mathrm{q}}$, which in turn implies $\underset{\sim}{\mathrm{q}} \in w_{2}^{1}$. This concludes the proof.

We are now in a position to take up

Theorem 3.2. (Uniqueness of solution to the traction problem for weak elastodynamic states). Let $\mathrm{R}$ be a regular locally star-shaped $\underline{\text { region and suppose: }}$

$$
\begin{aligned}
& \text { (a) } S^{\prime}=\left[\underset{\sim}{u^{\prime}}, \sigma_{\sim}^{\prime}\right] \in \varepsilon_{w}\left(\underset{\sim}{f}, \rho, \underset{\sim}{c} ; \bar{R} \times\left[0, t_{0}\right]\right), \\
& S^{\prime \prime}=\left[{\underset{\sim}{u}}^{\prime \prime}, \sigma_{\sim}^{\prime \prime}\right] \in \varepsilon_{\mathrm{w}}\left(\underset{\sim}{f}, \rho, \underset{\sim}{c} ; \overline{\mathrm{R}} \times\left[0, \mathrm{t}_{\mathrm{O}}\right]\right) . \\
& \text { ( } \beta) \text { For every } \varphi \in \hat{\mathcal{F}}\left(\overline{\mathrm{R}} \times\left[0, \mathrm{t}_{0}\right]\right) \text {, }
\end{aligned}
$$

$$
\begin{aligned}
& \mathrm{t}_{\mathrm{O}} \\
& \iint\left(\rho \dot{\varphi}_{i} \dot{\partial u}_{i}^{\prime}-\varphi_{i, j} \sigma_{i j}^{\prime}+\varphi_{i} f_{i}\right) d V d t+\int \rho(\underset{\sim}{x}) \varphi_{i} \underset{\sim}{(x, 0)} \stackrel{\circ}{v}_{i} \underset{\sim}{(x)} d V+\iint_{\sim} \varphi_{i} \stackrel{*}{s}_{i} d A d t=0, \\
& 0 \mathrm{R} \quad \mathrm{R} \quad 0 \partial \mathrm{R} \\
& \left.\int_{0}^{t_{R}} \int_{R}\left(\rho \dot{\varphi}_{i} \dot{\partial}_{i}^{\prime \prime}-\varphi_{i, j} \sigma_{i j}^{\prime \prime}+\varphi_{i} f_{i}\right) d v d t+\int_{R} \rho(\underset{\sim}{x}) \varphi_{i} \underset{\sim}{(x}, 0\right) \stackrel{\circ}{v}_{i} \underset{\sim}{(x)} d V+\int_{0}^{t_{0}} \int_{\partial R} \varphi_{i} \stackrel{*}{s}_{i} d A d t=0,
\end{aligned}
$$

whereas, 


$$
\left.\left.\lim _{t \rightarrow 0} \int_{R} \mid \underset{\sim}{u^{\prime}}(\underset{\sim}{x}, t)-\underset{\sim}{\operatorname{ur}} \underset{\sim}{x}\right)\left.\right|^{2} d V=0 \quad, \quad \lim _{t \rightarrow 0} \int_{R} \mid \underset{\sim}{u^{\prime \prime}}(\underset{\sim}{x}, t)-\underset{\sim}{\stackrel{o}{u}} \underset{\sim}{x}\right)\left.\right|^{2} d V=0,
$$

$\stackrel{\circ}{\sim}, \stackrel{\circ}{\sim}$ and $\stackrel{*}{\stackrel{*}{\mathrm{~S}} \text { being square-summable vector fields on }} \overline{\mathrm{R}}, \overline{\mathrm{R}}$ and $\partial \mathrm{R} \times\left[0, \mathrm{t}_{\mathrm{O}}\right]$, respectively.

In addition, as sume

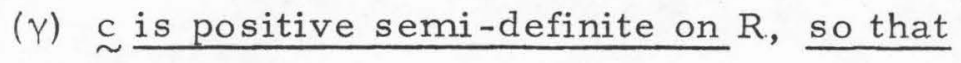

$$
c_{i j k \ell} \stackrel{(x)}{\sim} \gamma_{i j} \gamma_{k \ell} \geq 0 \text { for every } \underset{\sim}{x} \in R
$$

and for every symmetric two-tensor $\underset{\sim}{\mathcal{Y}}$ Then, almost everywhere,

$$
\stackrel{u}{\sim}^{\prime}={\underset{\sim}{u}}^{\prime \prime} \quad, \quad \sigma^{\prime}={\underset{\sim}{\sigma}}^{\prime \prime} \text { on } \overline{\mathrm{R}} \times\left[0, \mathrm{t}_{0}\right]
$$

Proof: Define a state $S=[\underset{\sim}{\mathrm{u}}, \underset{\sim}{\sigma}]$ through

$$
\underset{\sim}{\mathrm{u}}={\underset{\sim}{\mathrm{u}}}^{\prime}-\underline{\sim}_{\sim}^{\prime \prime}, \quad \underset{\sim}{\sigma}=\sigma^{\prime}-\sigma^{\prime \prime} \text { on } \overline{\mathrm{R}} \times\left[0, \mathrm{t}_{\mathrm{o}}\right] .
$$

Then, according to Hypotheses $(\alpha),(\beta)$ and Definition 3.1,

$$
S=[\underset{\sim}{\sim} \underset{\sim}{\sigma}] \in \varepsilon_{\mathrm{w}}\left(\underset{\sim}{\sim}, \rho, \underset{\sim}{\mathrm{c}} ; \overline{\mathrm{R}} \times\left[0, \mathrm{t}_{\mathrm{o}}\right]\right),
$$

$$
\int_{0}^{t_{0}} \int_{R}\left(\rho \dot{\varphi}_{i} \dot{\partial} u_{i}-\varphi_{i, j} \sigma_{i j}\right) d V d t=0 \text { for every } \underset{\sim}{\varphi} \in \hat{G}\left(\bar{R} \times\left[0, t_{o}\right]\right),
$$

$$
\lim _{t \rightarrow 0} \int_{R}|\underset{\sim}{u}(x, t)|^{2} d V=0
$$

We show first that (C) holds also for each $\underset{\sim}{\varphi} \in \frac{\sqrt{k}}{*}\left(\bar{R} \times\left[0, t_{0} 7\right)\right.$, provided $\dot{\varphi}_{i}, \varphi_{i, j}$ are replaced by $\dot{\partial}_{i}, \partial_{j} \varphi_{i} ;$ i. e. ,

$$
\int_{0}^{t_{0}} \int_{R}\left(\rho \dot{\partial} \varphi_{i} \dot{\partial} u_{i}-\partial_{j} \varphi_{i} \sigma_{i j}\right) d V d t=0 \text { for every } \underset{\sim}{\varphi} \underset{\sim}{\stackrel{*}{*}}\left(\bar{R} \times\left[0, t_{o}\right]\right) .
$$


Indeed, let $\underset{\sim}{\rho \in} \in\left(\bar{R} \times\left(\bar{R} \times\left[0, t_{0}\right]\right)\right.$. Then (see (iii) of Definition 3.2), there exists a sequence $\left\{\stackrel{\varphi}{n}^{n}\right\}$ of vector fields such that

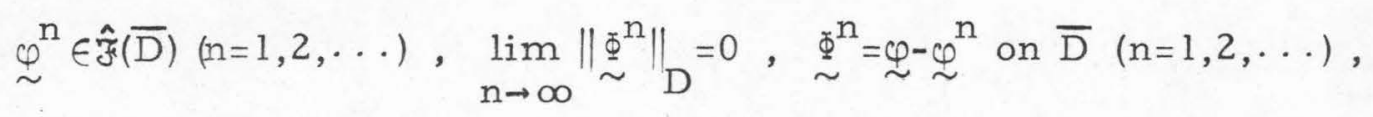

where $D=R \times\left(0, t_{0}\right)$. Next, set

$$
\Delta=\int_{0}^{t_{0}} \int_{R}\left(\rho \dot{\partial} \varphi_{i} \dot{\partial} u_{i}-\partial_{j} \varphi_{i} \sigma_{i j}\right) d v d t
$$

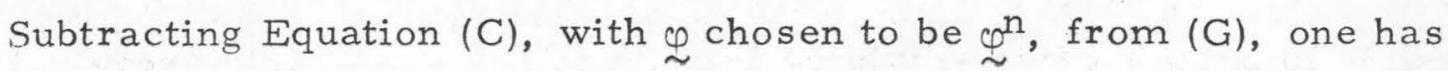

$$
\Delta=\int_{0}^{t_{0}} \int_{R}\left(\rho \partial \Phi_{i}^{n} \dot{\partial u}_{i}-\partial_{j} \Phi_{i}^{n} \sigma_{i j}\right) d V d t \quad(n=1,2, \cdots),
$$

where $\underset{\sim}{\mathrm{n}}$ is given by the last of $(\mathrm{F})$. From $(\mathrm{H})$ and the Schwarz inequality for $\aleph_{2}$-functions ${ }^{1}$, one draws

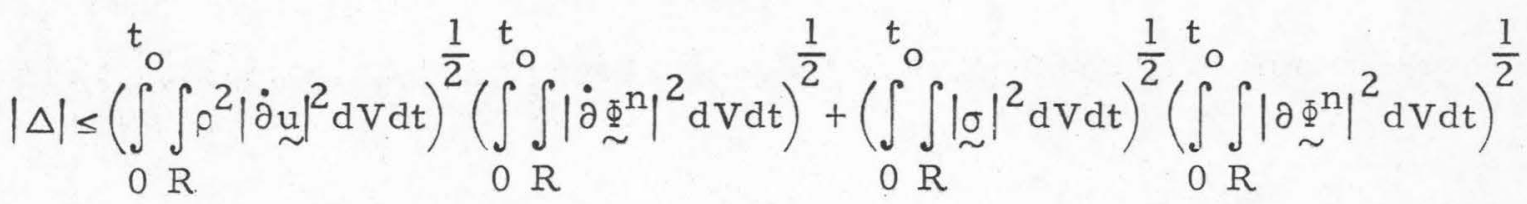
for every $n(n=1,2, \cdots)$. Hence, $\Delta=0$ by virtue of the second of $(F)$ and (3.3), so that $(E)$ now follows from (G).

Our next objective is to deduce ${ }^{2}$ the desired results from (E). To accomplish this, choose $t_{1} \in\left[0, t_{0}\right)$ and set

${ }^{1}$ We define the inner product of two $\mathrm{n}^{\text {th }}$-order tensor-valued functions in $\mathcal{\delta}_{2}\left(\bar{R} \times\left[0, t_{0}\right]\right)$ to be the integral over $R \times\left(0, t_{0}\right)$ of their fully contracted outer product.

2 The remainder of the proof is suggested by an argument due to Ladyzhenskaya [5] (p. 72). 


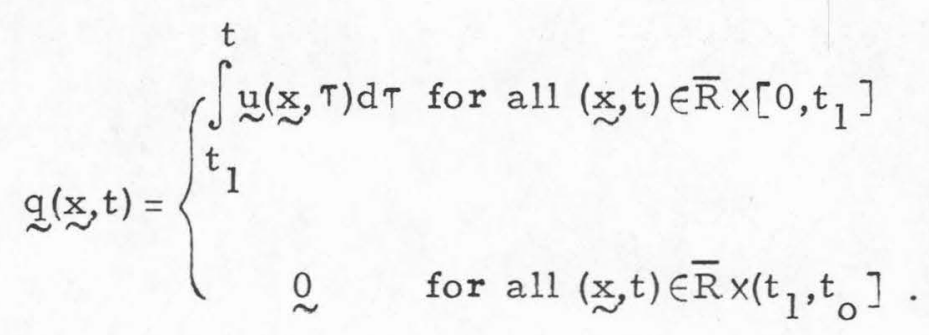

Then, according to Lemma 3.1,

$$
\begin{aligned}
& \mathrm{q} \in \underset{\sim}{*}(\overline{\mathrm{R}} \times[0, \mathrm{t}, 7), \\
& \dot{\partial} \underset{\sim}{q}(x, t)=\left\{\begin{array}{cl}
\underset{\sim}{u}(\underset{\sim}{x}, t) & \text { for all } \underset{\sim}{(x, t)} \in \bar{R} \times\left[0, t_{1}\right] \\
\underset{\sim}{0} & \text { for all } \underset{\sim}{(x, t)} \in \bar{R} \times\left(t_{1}, t t_{0}\right],
\end{array}\right. \\
& \partial \underset{\sim}{\mathrm{q}}(\underset{\sim}{\mathrm{x}}, \mathrm{t})=\left\{\begin{array}{cc}
\int_{\mathrm{t}}^{\mathrm{t}} \partial \underset{\sim}{\mathrm{u}}(\underset{\sim}{\mathrm{x}}, \tau) \mathrm{d} \tau \text { for all } \underset{\sim}{(\underset{\sim}{x}, t) \in \bar{R} \times\left[0, t_{1}\right]} \\
\underset{\sim}{0} & \text { for all } \underset{\sim}{(x, t)} \in \overline{\mathrm{R}} \times\left(t_{1}, t_{0}\right] .
\end{array}\right.
\end{aligned}
$$

Because of $(K)$ and $(L)$, and since $\underset{\sim}{u} \in \log _{2}^{1}\left(\bar{R} \times\left[0, t_{0}\right]\right)(c f .,(B)$ and Definition 3.1 ), $\underset{\sim}{q}$ and $\underset{\sim}{q}$ have square-integrable generalized timederivatives on $\bar{R} \times\left[0, t_{1}\right]$, which are given by

$$
\dot{\partial}(\underset{\sim}{q})=\dot{\partial} \underset{\sim}{u}, \quad \dot{\partial}(\partial \underset{\sim}{q})=\partial \underset{\sim}{u} \text { on } \bar{R} \times\left[0, t_{1}\right] .
$$

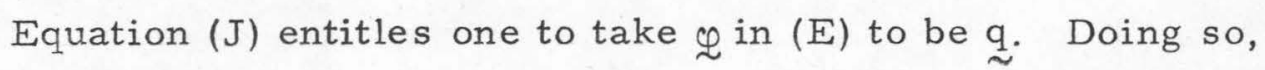
thereafter substituting for $\underset{\sim}{\sigma}$ from (3.5) and for $\underset{\sim}{\dot{u}}, \partial \underset{\sim}{u}$ from $(M)$, one arrives at

$$
\int_{0}^{t_{R}} \int_{R}\left[\rho \dot{\partial} q_{i} \dot{\partial}\left(\dot{\partial} q_{i}\right)-c_{i j k \ell} \dot{\partial}\left(\partial_{\ell} q_{k}\right) \partial_{j} q_{i}\right] d v d t=0
$$

On the other hand, the symmetry-relations (1.5) for $\underset{\sim}{c}$ together with the product rule for generalized time-differentiation gives 


$$
c_{i j k \ell} \dot{\partial}\left(\partial_{\ell} q_{k}\right) \partial_{j} q_{i}=\frac{1}{2} \dot{\partial}\left(c_{i j k \ell} \partial_{j} q_{i} \partial_{\ell} q_{k}\right) \text { on } \bar{R} \times\left[0, t_{l}\right] \text {. }
$$

From (N) and (P) follows

$$
\int_{0}^{t} \int_{R}^{1} \dot{\partial}\left(\rho \dot{\partial} q_{i} \dot{\partial} q_{i}-c_{i j k \ell} \partial{ }_{j} q_{i} \partial_{l} q_{k}\right) d v d t=0
$$

Now interchange the order of integration in (Q) and carry out the timeintegration $^{1}$, bearing in mind that $\underset{\sim}{\mathrm{q}}(\cdot, 0)$ and $\underset{\sim}{\mathrm{q}}\left(\cdot, \mathrm{t}_{1}\right)$ vanish almost everywhere on $\bar{R}$ in view of $(K)$, (D) and (L). Consequently,

$$
\left.\int_{R}\left(\rho(\underset{\sim}{x}) \dot{\partial} q_{i}\left(\underset{\sim}{\sim}, t_{1}\right) \dot{\partial} q_{i} \underset{\sim}{(x, t}\right)+c_{i j k \ell} \partial_{j} q_{i} \underset{\sim}{(x, 0)} \partial_{l} q_{k} \underset{\sim}{(x, 0)}\right) d V=0 .
$$
R

Equation (S) and Hypothesis $(Y)$, along with $(K)$, imply

$$
\int_{R} \rho(\underset{\sim}{x}) u_{\sim}^{2}\left(\underset{\sim}{x}, t_{1}\right) d V=0
$$

or, since $\rho$ is positive and $t_{1} \in\left[0, t_{0}\right)$ was chosen arbitrarily,

$$
\int_{R}|\underset{\sim}{u}(x, t)|^{2} d V=0 \text { for every } t \in\left[0, t_{0}\right) \text {. }
$$

Equations (T) and (A) yield the first of (3.13).

Finally, (A) and the first of (3.13), together with the formula for the generalized gradient of $\underset{\sim}{u}\left(c f_{.},(3.2)\right)$, assure that $\underset{\sim}{u}$ vanishes almost everywhere on $\bar{R} \times\left[0, t_{0}\right]$. Therefore, by (3.5), $\sigma$ vanishes almost everywhere on $\bar{R} \times\left[0, t_{0}\right]$, whence the second of (3.13) holds true. This completes the proof.

Among the types of singularities admissible in solutions to dynamical elasticity problems that are covered by Theorem 3.2 are, ${ }^{1}$ Recall the last of (3.7). 
besides finite jump discontinuities in the stress field, square-integrable isolated stress-infinities, such as those arising in connection with the focusing of elastic waves. The formulation of the traction problem given here, however, precludes the possibility that the displacement field itself undergoes a finite discontinuity across some surface, since such a singularity cannot occur in a function belonging to $\mathrm{w}_{2}^{1}$ (see, for example, Smirnov [16], Section 110). It may be well to emphasize

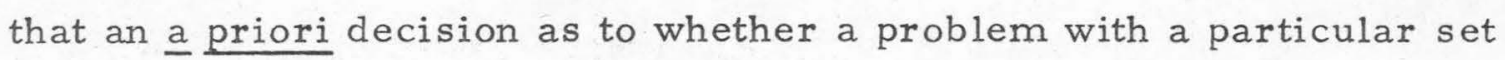
of data is covered by Theorem 3.2 would require an existence theorem appropriate to the traction problem for weak elastodynamic states. This existence issue is beyond the scope of the present investigation. Finally, we observe that the regularity hypotheses on $\rho$ and $\underset{\sim}{c}$ could easily be relaxed to accommodate discontinuities in material properties. We conclude this section with a specialization of Theorem 3.2 to piecewise regular elastodynamic states. Toward this end, we introduce

Lemma 3.2. Let $\mathrm{R}$ be a regular region and

$$
\left.S=[\underset{\sim}{u} \underset{\sim}{\sigma}] \in \varepsilon_{\mathrm{p}} \underset{\sim}{(f}, \rho, \mathrm{c} ; \overline{\mathrm{R}} \times\left[0, \mathrm{t}_{\mathrm{o}}\right]\right) .
$$

Then,

$$
S \in \varepsilon_{w}\left(\stackrel{f}{\sim}, \rho, c ; \sim \bar{R} \times\left[0, t_{0}\right]\right),
$$

and, for every $\underline{\sim} \in \hat{\mathfrak{F}}\left(\overline{\mathrm{R}} \times\left[0, \mathrm{t}_{\mathrm{o}}\right]\right)$,

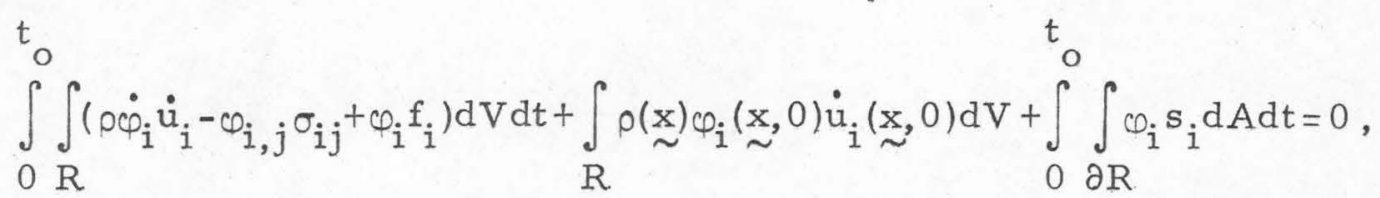

$\stackrel{\mathrm{s}}{\sim}$ being the traction vector of $\mathrm{S}$ on $\partial \mathrm{R}$. 
Proof: Let $\left\{D_{1}, D_{2}, \ldots, D_{m}\right\}$ be the regular partition of $R \times\left(0, t_{0}\right)$ appropriate to $\mathrm{S}$ (see Definition 2.4). We verify first that

$$
\underset{\sim}{f} \in \mathcal{L}_{2}\left(\overline{\mathrm{R}} \times\left[0, t_{0}\right]\right) \quad, \quad \underset{\sim}{u} \in w_{2}^{1}\left(\overline{\mathrm{R}} \times\left[0, t_{0}\right]\right) .
$$

The first of (A) is immediate from (i) in Definition 2.4. As for the second of $(A)$, fix $j(j=1,2,3)$ and let $\underset{\sim j}{\mathrm{e}}$ be the unit base vector in the $x_{j}$-direction. Choose $\underset{\sim}{\varphi} \in \hat{C}^{1}\left(\bar{R} \times\left[0, t_{0}\right]\right)$ and introduce a vector field ${\underset{\sim}{p}}^{\prime}$ and a scalar field $\mathrm{q}^{\prime}$ through

$$
\stackrel{p^{\prime}}{\sim}\left(\varphi_{i} u_{i}\right) \underset{\sim j}{e} \quad, \quad q^{\prime}=\varphi_{i} u_{i} \text { on } \bar{R} \times\left[0, t_{0}\right] .
$$

From (B), Definition 2.4, and the assumed regularity of $\varrho$ follows

$$
\underline{\mathrm{p}}^{\prime} \in \mathrm{C}^{1}\left(\overline{\mathrm{D}}_{\mathrm{k}}\right) \quad, \quad \mathrm{q}^{\prime} \in \mathrm{C}^{1}\left(\overline{\mathrm{D}}_{\mathrm{k}}\right) \quad(\mathrm{k}=1, \cdots, \mathrm{m}) .
$$

Thus, we may apply Lemma 2.1 first with $\underset{\sim}{\mathrm{p}}=\mathrm{p}_{\sim}^{\prime}, \mathrm{q}=0$ and then with $\underset{\sim}{\mathrm{p}}=0$, $\mathrm{q}=\mathrm{q}^{\prime}$. Doing so and subsequently appealing to (B) and the fact that $\stackrel{\varphi}{\sim}(\cdot, 0), \underset{\sim}{\varphi}\left(\cdot, t_{0}\right)$ vanish on $\bar{R}$, we arrive at

$$
\begin{aligned}
& \int_{0}^{t_{0}} \int_{R_{t}^{k}}\left(\varphi_{i} u_{i}\right), j d v d t=\int_{0}^{t_{0}} \int_{\partial R_{t}^{k}} n_{j}^{k} \varphi_{i} u_{i} d A d t, \\
& \int_{0}^{t_{0}} \int_{t}^{k} \frac{\partial}{\partial t}\left(\varphi_{i} u_{i}\right) d V d t=-\int_{0}^{t_{0}} \int_{\partial R_{t}^{k}} x^{k} \varphi_{i} u_{i} d A d t,
\end{aligned}
$$

for each $k(1 \leq k \leq m)$, where $R_{t}^{k}\left(0 \leq t \leq t_{0}\right)$ is the piecewise smooth family of regions that sweeps out $D_{k}$. Since $\varphi_{i} u_{i}$ is continuous on $\bar{R} \times\left[0, t_{0}\right]$ and $\underset{\sim}{\varphi}$ vanishes on $\partial \mathrm{R} \times\left[0, \mathrm{t}_{\mathrm{O}}\right]$, summing $(\mathrm{D})$ and $(\mathrm{E})$ over $\mathrm{k}$ yields ${ }^{1}$

${ }_{\text {Recall that }}\left\{D_{1}, D_{2}, \cdots, D_{m}\right\}$ partitions $R \times\left(0, t_{0}\right)$. 


$$
\int_{0}^{t_{0}} \int_{R}\left(\varphi_{i} u_{i}\right), j d v d t=0, \quad \int_{0}^{t_{0}} \int_{R} \frac{\partial}{\partial t}\left(\varphi_{i} u_{i}\right) d V d t=0,
$$

which in turn implies that $\underset{\sim}{\sim}$ and $\underset{\sim}{\dot{u}}$ are the generalized gradient and the generalized time-derivative of $\underset{\sim}{u}$ on $\bar{R} \times\left[0, t_{0}\right]$. Since, by Definition 2.4, $\nabla \underset{\sim}{u}$ and $\underset{\sim}{\dot{u}}$ lie in $\mathcal{S}_{2}\left(\bar{R} \times\left[0, t_{0}\right]\right)$, the first of $(A)$ follows.

We turn next to the proof of (3.14). For this purpose, let $\mathrm{k}(1 \leq \mathrm{k} \leq \mathrm{m})$ be a fixed integer, choose $\varphi \in \hat{\mathcal{F}}\left(\overline{\mathrm{R}} \times\left[0, \mathrm{t}_{0}\right]\right)$, and define a vector field $\underset{\sim}{\mathrm{p}}$ and a scalar field $\mathrm{q}$ by means of

$$
\begin{aligned}
& \mathrm{p}_{\mathrm{j}}=-\varphi_{\mathrm{i}} \sigma_{i j} \text { in } \mathrm{D}_{\mathrm{k}}, \quad \mathrm{p} \in \mathrm{C}^{1}\left(\overline{\mathrm{D}}_{\mathrm{k}}\right), \\
& \mathrm{q}=\rho \varphi_{i} \dot{\mathrm{u}}_{\mathrm{i}} \text { in } \mathrm{D}_{\mathrm{k}}, \quad \mathrm{q} \in \mathrm{C}^{1}\left(\overline{\mathrm{D}}_{\mathrm{k}}\right) .
\end{aligned}
$$

A straightforward computation, involving (G) and the stress equations of motion (1.7), leads to

$$
p_{j, j}+\dot{q}=\rho \dot{c}_{i} \dot{u}_{i}-\varphi_{i, j} \sigma_{i j}+\varphi_{i} f_{i} \text { in } D_{k}
$$

Applying Lemma 2.1 to $\underset{\sim}{\mathrm{p}}, \mathrm{q}$ on $\overline{\mathrm{D}}_{\mathrm{k}}$ and arguing as in the proof of Theorem 2.2-again using the jump conditions (2.8)-we deduce (3.14).

But (3.14) implies (3.6). Moreover, (3.5) follows at once from (1.6) so that, because of (A),

$$
\mathrm{S} \in \varepsilon_{\mathrm{W}}\left(\underset{\sim}{\mathrm{f}}, \rho, \mathrm{c} ; \bar{\sim} \times\left[0, \mathrm{t}_{\mathrm{o}}\right]\right) .
$$

The proof is now complete.

It is worth noting that one can establish the following converse of Lemma 3.2. Suppose $\mathrm{R}$ is a regular region and

$$
\begin{gathered}
S=[\underset{\sim}{\mathrm{u}} \underset{\sim}{\sigma}] \in \mathcal{E}_{\mathrm{w}}\left(\underset{\sim}{f}, \rho, \mathrm{c} ; \overline{\mathrm{R}} \times\left[0, \mathrm{t}_{\mathrm{o}}\right]\right), \\
\underset{\sim}{\mathrm{u}} \in \mathbb{C}\left(\overline{\mathrm{R}} \times\left[0, \mathrm{t}_{\mathrm{o}}\right]\right), \quad \underset{\sim}{\mathrm{f}} \in \mathbb{C}\left(\overline{\mathrm{R}} \times\left[0, \mathrm{t}_{\mathrm{o}}\right]\right) .
\end{gathered}
$$


Assume in addition that there exists a regular partition $\left\{\mathrm{D}_{1}, \mathrm{D}_{2}, \cdots, \mathrm{D}_{\mathrm{m}}\right\}$ of $R \times\left(0, t_{0}\right)$ such that, for each $k(1 \leq k \leq m)$, S coincides on $D_{k}$ with a state $S^{k}=\left[\underset{\sim}{u}, \sigma^{k}\right]$ that is defined on $\bar{D}_{k}$ and satisfies

$$
\underset{\sim}{\mathrm{u}} \in \mathrm{C}^{2}\left(\overline{\mathrm{D}}_{\mathrm{k}}\right), \underset{\sim}{\sigma^{\mathrm{k}}} \in \mathrm{C}^{1}\left(\overline{\mathrm{D}}_{\mathrm{k}}\right)
$$

Then,

$$
S \in \mathcal{E}_{\mathrm{p}}\left(\underset{\sim}{f}, \rho, \underset{\sim}{c} ; \overline{\mathrm{R}} \times\left[0, \mathrm{t}_{\mathrm{o}}\right]\right) .
$$

The result just mentioned provides an alternative derivation of the classical jump relations (2.8).

Theorem 3.3. (Uniqueness of solution to the traction problem for piecewise regular elastodynamic states). Let $R$ be a regular, locally star-shaped region. Then the conclusion in Theorem 2.3 follows from Hypotheses $(\alpha),(\gamma)$ and $(\delta)$ alone; that is, in the absence of $(\beta)$.

Proof: Hypothesis $(\alpha)$ in Theorem 2.3, Lemma 3.2, and Definition 3.1 imply

$$
s \in \mathcal{E}_{\mathrm{w}}\left(\underset{\sim}{0}, \rho, \mathrm{c} ; \overline{\mathrm{R}} \times\left[0, \mathrm{t}_{\mathrm{o}}\right]\right) .
$$

Moreover, Hypothesis ( $(y)$ and Equation (3.14) applied to S yield

$$
\int_{0}^{t_{0}} \int_{R}\left(\rho \dot{\varphi}_{i} \dot{u}_{i}-\varphi_{i, j} \sigma_{i j}\right) d V d t=0 \text { for every } \underset{\sim}{\varphi \in} \hat{\dot{G}}\left(\bar{R} \times\left[0, t_{0}\right]\right) \text {, }
$$

$$
\underset{\sim}{u}(\cdot, 0)=0 \text { on } \bar{R} .
$$

Equations (A), (B) and (C), in conjunction with Theorem 3.2, necessitate that

$$
\underset{\sim}{\mathrm{u}}=\underset{\sim}{0}, \quad \underset{\sim}{\sigma}=0 \quad \text { almost everywhere on } \overline{\mathrm{R}} \times\left[0, \mathrm{t}_{\mathrm{O}}\right] \text {. }
$$


But $\underset{\sim}{u}$ is continuous on $\bar{R}$, and $\underset{\sim}{\sigma}$ is continuous on $\bar{R} \times\left[0, t_{0}\right]-\bar{A}$, so that

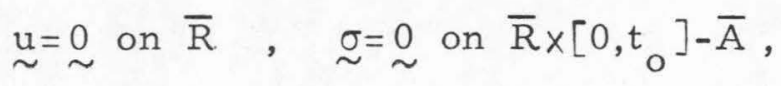

and the proof is complete.

We have thus obtained a modified version of Theorem 2.3 which is free from the artificial Assumption $(\beta)$, but which involves a slight change in the hypothesis on the underlying spatial domain $\mathrm{R}$. The reason for the presence of $(\beta)$ in Theorem 2.3 and its absence in Theorem 3.3 is that while the difference in two weak elastodynamic states is obviously itself such a state, the analogous claim for piecewise regular elastodynamic states cannot be taken for granted. In conclusion, we remark that a counterpart of Theorem 3.2 for the first problem with homogeneous boundary conditions can be established through a scheme very much like the one used here to cope with the second problem ${ }^{1}$. If one then adds to Definition 3.2 the requirement that the displacement field be continuous on $\bar{R} \times\left[0, t_{0}\right]$, an analogous uniqueness theorem for inhomogeneous surface data can also be obtained, provided the region $\mathrm{R}$ satisfies certain regularity conditions beyond those assumed in this investigation. Similarly, it is possible to generalize Theorem 3.2 to the mixed problem of classical elastodynamics ${ }^{2}$.

1 Ladyzhenskaya [5] has treated the corresponding uniqueness issue for a single, second-order hyperbolic equation.

${ }^{2}$ See the paper by Dafermos [9] on linear dynamical thermoelasticity in which the uniqueness of the "generalized" solution to a mixed boundary-initial value problem for homogeneous boundary data is proved. 


\section{References}

[1] F. Neumann, Vorlesungen über die Theorie der Elasticität der fester Körper und des Lichtäthers, B. G. Teubner, Leipzig, 1885.

[2] C. Truesdell and R. A. Toupin, The classical field theories, Handbuch der Physik, vol. III/1, Springer, Berlin, 1960.

[3] M. E. Gurtin, The linear theory of elasticity, unpublished manuscript to appear in the Handbuch der Physik, Springer, Berlin.

[4] S. L. Sobolev, Application of functional analysis in mathematical physics, Translations of Mathematical Monographs, vol. 7, American Mathematical Society, Providence, Rhode Island, 1963.

[5] O. A. Ladyzhenskaya, The mixed problem for a hyperbolic equation, State Publishing House for Technical-Theoretical Literature, Moscow, 1953 (In Russian).

[6] R. J. Knops and L. E. Payne, Uniqueness in classical elastodynamics, Archive for Rational Mechanics and Analysis, 27 (1968), 349.

[7] R. J. Knops and L. E. Payne, Uniqueness theorems in linear elasticity, Springer, Berlin, 1971.

[8] L. Brun, Sur 1'unicité en thermoélasticité dynamique et diverses expressions analogues a la formule de Clapeyron, Comptes Rendus, Académie des Sciences, 261 (1965), 2584.

[9] C. M. Dafermos, On the existence and asymptotic stability of solutions to the equations of linear thermoelasticity, Archive for Rational Mechanics and Analysis, 29 (1968), 241.

[10] K. O. Friedrichs, Symmetric positive linear differential equations, Communications on Pure and Applied Mathematics, $11(1958), 333$.

[11] O. D. Kellogg, Foundations of potential theory, Frederick Ungar, New York, 1929.

[12] L. T. Wheeler and E. Sternberg, Some theorems in classical elastodynamics, Archive for Rational Mechanics and Analysis, 31 (1968), 51 . 
[13] L. T. Wheeler, Some results in the linear dynamical theory of anisotropic elastic solids, Quarterly of Applied Mathematics, 28 (1970), 91 .

[14] H. B. Keller, Propagation of stress discontinuities in inhomogeneous elastic media, SIAM Review, 6 (1964), 356.

[15] R. C. Buck and E. F. Buck, Advanced calculus, McGraw-Hill, New York, 1965.

[16] V. I. Smirnov, Integration and functional analysis, A Course of Higher Mathematics, vol. V, Addison-Wesley, Reading, Massachusetts, 1964.

[17] K. O. Friedrichs, The identity of weak and strong extensions of differential operators, Transactions of the American Mathematical Society, 55 (1944), 132.

[18] S. Agmon, Lectures on elliptic boundary value problems, D. Van Nostrand, Princeton, New Jersey, 1965.

[19] H. Bremermann, Distributions, complex variables, and Fourier transforms, Addison-Wesley, Reading, Massachusetts, 1965.

[20] A. Friedman, Partial differential equations of parabolic type, Prentice-Hall, Englewood Cliffs, New Jersey, 1964.

[21] E. J. McShane and L. A. Botts, Real analysis, D. Van Nostrand, Princeton, New Jersey, 1959. 